\title{
A Central Limit Theorem for Realised Power and Bipower Variations of Continuous Semimartingales
}

\author{
Ole E. BARNDORFF-NIELSEN ${ }^{1}$, Svend Erik GRAVERSEN ${ }^{2}$,
} Jean $\mathrm{JACOD}^{3}$, Mark PODOLSKIJ ${ }^{4 \star}$, and Neil SHEPHARD ${ }^{5}$

1 Dept. of Mathematical Sciences, University of Aarhus, Ny Munkegade, DK-8000 Aarhus C, Denmark, (e-mail: oebn@imf.au.dk)

2 Dept. of Mathematical Sciences, University of Aarhus, Ny Munkegade, DK-8000 Aarhus C, Denmark, (e-mail:matseg@imf.au.dk)

3 Laboratoire de Probabilités et Modèles Aléatoires (CNRS UMR 7599) Université P. et M. Curie, 4 Place Jussieu, 75252 - Paris Cedex, France. (e-mail: jj@ccr.jussieu.fr)

4 Ruhr University of Bochum, Dept. of Probability and Statistics, Universitätstrasse 150, 44801 Bochum, Germany, (e-mail:podolski@cityweb.de)

${ }^{5}$ Nuffield College, Oxford OX1 1NF, UK, (e-mail: neil.shephard@nuf.ox.ac.uk)

Summary. Consider a semimartingale of the form $Y_{t}=Y_{0}+\int_{0}^{t} a_{s} d s+\int_{0}^{t} \sigma_{s-} d W_{s}$, where $a$ is a locally bounded predictable process and $\sigma$ (the "volatility") is an adapted right-continuous process with left limits and $W$ is a Brownian motion. We consider the realised bipower variation process

$$
V(Y ; r, s)_{t}^{n}=n^{\frac{r+s}{2}-1} \sum_{i=1}^{[n t]}\left|Y_{\frac{i}{n}}-Y_{\frac{i-1}{n}}\right|^{r}\left|Y_{\frac{i+1}{n}}-Y_{\frac{i}{n}}\right|^{s},
$$

where $r$ and $s$ are nonnegative reals with $r+s>0$. We prove that $V(Y ; r, s)_{t}^{n}$ converges locally uniformly in time, in probability, to a limiting process $V(Y ; r, s)_{t}$ (the "bipower variation process"). If further $\sigma$ is a possibly discontinuous semimartingale driven by a Brownian motion which may be correlated with $W$ and by a Poisson random measure, we prove that $\sqrt{n}\left(V(Y ; r, s)^{n}-V(Y ; r, s)\right)$ converges in law to a process which is the stochastic integral with respect to some other Brownian motion $W^{\prime}$, which is independent of the driving terms of $Y$ and $\sigma$. We also provide a multivariate version of these results, and a version in which the absolute powers are replaced by smooth enough functions.

Key words: Central limit theorem, quadratic variation, bipower variation.

* This author has been partially supported by the DYNSTOCH Research Training Network, and the financial support of the Deutsche Forschungsgemeinschaft (SFB 475, "Reduction of complexity in multivariate data structures") is gratefully acknowledged. 


\section{Introduction}

For a wide class of real-valued processes $Y$, including all semimartingales, the "approximate (or, realised) quadratic variation processes"

$$
V(Y ; 2)_{t}^{n}=\sum_{i=1}^{[n t]}\left(Y_{\frac{i}{n}}-Y_{\frac{i-1}{n}}\right)^{2}
$$

where $[x]$ denotes the integer part of $x \in \mathbf{R}_{+}$, converge in probability, as $n \rightarrow \infty$ and for all $t \geq 0$, towards the quadratic variation process $V(Y ; 2)_{t}$, usually denoted by $[Y, Y]_{t}$.

This fact is basic in the "general theory of processes" and is also used in a large variety of more concrete problems, and in particular for the statistical analysis of the process $Y$ when it is observed at the discrete times $i / n: i=0,1, \ldots$ (sometimes $V(Y ; 2)_{t}^{n}$ is called the "realised" quadratic variation, since it is explicitly calculable on the basis of the observations). In that context, in addition to the convergence in probability one is interested in the associated CLT (Central Limit Theorem), which says that the $\sqrt{n}\left(V(Y ; 2)_{t}^{n}-V(Y ; 2)_{t}\right)$ 's converge in law, as processes, to a non-trivial limiting process. Of course, for the CLT to hold we need suitable assumptions on $Y$. This type of tool has been used very widely in the study of the statistics of processes in the past twenty years. References include, for example, the review paper [10] in the statistics of processes and [1], [2], [3], [6] in financial econometrics. [2] provides a review of the literature in econometrics on this topic.

Now, when $Y$ describes some stock price, with a stochastic volatility possibly having jumps, a whole new class of processes extending the quadratic variation has been recently introduced, and named "bipower variation processes": let $r, s$ be nonnegative numbers. The realised bipower variation process of order $(r, s)$ is the increasing processes defined as:

$$
V(Y ; r, s)_{t}^{n}=n^{\frac{r+s}{2}-1} \sum_{i=1}^{[n t]}\left|Y_{\frac{i}{n}}-Y_{\frac{i-1}{n}}\right|^{r}\left|Y_{\frac{i+1}{n}}-Y_{\frac{i}{n}}\right|^{s},
$$

with the convention $0^{0}=1$. Clearly $V(Y ; 2)^{n}=V(Y ; 2,0)^{n}$. The bipower variation process of order $(r, s)$ for $Y$, denoted by $V(Y ; r, s)_{t}$, is the limit in probability, if it exists for all $t \geq 0$, of $V(Y ; r, s)_{t}^{n}$. It has been introduced in [4] and [5], where it is shown that the bipower variation processes exist for all nonnegative indices $r, s$ as soon as $Y$ is a continuous semimartingale of "Itô type" with smooth enough coefficients. These papers also contain a version of the associated CLT under somewhat restrictive assumptions and when $r=s=1$.

The aim of this paper is mainly to investigate the CLT, and more precisely to give weaker conditions on $Y$ which ensure that it holds and which cover 
most concrete situations of interest, and also to precisely describe the limiting process. We prove the existence of the bipower variation process for a wide class of continuous semimartingales (extending the results of [4] and [5]). We establish the CLT in a slightly more restricted setting. The restriction is that the volatility of $Y$ (that is, the coefficient in front of the driving Wiener process for $Y$ ) is a semimartingale driven by a Lévy process, or more generally by a Wiener process (possibly correlated with the one driving $Y$ ) and a Poisson random measure.

We also investigate the multidimensional case, when $Y=\left(Y^{j}\right)_{1 \leq j \leq d}$ is $d-$ dimensional. It is then natural to replace (1.2) by the realised "cross-bipower variation processes":

$$
V\left(Y^{j}, Y^{k} ; r, s\right)_{t}^{n}=n^{\frac{r+s}{2}-1} \sum_{i=1}^{[n t]}\left|Y_{\frac{i}{n}}^{j}-Y_{\frac{i-1}{n}}^{j}\right|^{r}\left|Y_{\frac{i+1}{n}}^{k}-Y_{\frac{i}{n}}^{k}\right|^{s} .
$$

We state the results in Section 2, and the proofs are given in the other sections. The reader will notice that we replace the powers like $\left|Y_{\frac{i}{n}}-Y_{\frac{i-1}{n}}\right|^{r}$ in (1.2) by an expression of the form $g\left(\sqrt{n}\left(Y_{\frac{i}{n}}-Y_{\frac{i-1}{n}}\right)\right)$ for a suitable function $g$ : this can prove useful for some applications, and it is indeed a simplification rather than a complication for the proof itself. Written in this way, our results also extend some of the results of Becker in [7], and of the unpublished paper $[8]$.

It is also worth observing that, apart from the notational complexity, the proofs when $r>0$ and $s>0$ are not really more difficult than when $r>0$ and $s=0$, that is when we have only one power in (1.2). That means that, obviously, the same types of results would hold for the "realised multipower variation processes" which are defined by

$$
\begin{aligned}
& V\left(Y^{j_{1}}, \ldots, Y^{j_{N}} ; r_{1}, \ldots, r_{N}\right)_{t}^{n} \\
& \quad=n^{\frac{r_{1}+\ldots+r_{N}}{2}-1} \sum_{i=1}^{[n t]}\left|Y_{\frac{i}{n}}^{j_{1}}-Y_{\frac{i-1}{n}}^{j_{1}}\right|^{r_{1}} \ldots\left|Y_{\frac{i+N-1}{n}}^{j_{N}}-Y_{\frac{i+N-2}{n}}^{j_{N}}\right|^{N_{N}},
\end{aligned}
$$

for any choice of $r_{i} \geq 0$ and any fixed $N$. We do not prove those more general results here, but simply state the results.

\section{Statement of results}

We start with a filtered space $\left(\Omega, \mathcal{F},\left(\mathcal{F}_{t}\right)_{t \geq 0}, \mathbf{P}\right)$, on which are defined various processes, possibly multidimensional: so we systematically use matrix and product-matrices notations. The transpose is denoted by *, all norms are denoted by $\|$.$\| . We denote by \mathcal{M}_{d, d^{\prime}}$ the set of all $d \times d^{\prime}$-matrices, and by $\mathcal{M}_{d, d^{\prime}, d^{\prime \prime}}$ the set of all arrays of size $d \times d^{\prime} \times d^{\prime \prime}$, and so on. For any process $X$ we write $\Delta_{i}^{n} X=X_{i / n}-X_{(i-1) / n}$. 
Our basic process is a continuous $d$-dimensional semimartingale $Y=$ $\left(Y^{i}\right)_{1 \leq i \leq d}$. We are interested in the asymptotic behavior of all finite families of processes of type (1.3), that is for all $j, k \in\{1, \ldots, d\}$ and all finite families of pairs $(r, s)$. So in order to simplify notation (which will nevertheless remain quite complicated, sorry for that !), we introduce the following processes:

$$
X^{n}(g, h)_{t}=\frac{1}{n} \sum_{i=1}^{[n t]} g\left(\sqrt{n} \Delta_{i}^{n} Y\right) h\left(\sqrt{n} \Delta_{i+1}^{n} Y\right),
$$

where $g$ and $h$ are two maps on $\mathbf{R}^{d}$, taking vakues in $\mathcal{M}_{d_{1}, d_{2}}$ and $\mathcal{M}_{d_{2}, d_{3}}$ respectively. So $X^{n}(g, h)_{t}$ takes its values in $\mathcal{M}_{d_{1}, d_{3}}$. Note that, letting

$$
f_{j, r}(x)=\left|x^{j}\right|^{r}
$$

we have $V\left(Y^{j}, Y^{k} ; r, s\right)^{n}=X^{n}\left(f_{j, r}, f_{k, s}\right)$, and any finite family of processes like in (1.3) is a process of the type (2.1) with the components of $g$ and $h$ being the various $f_{j, r}$.

\subsection{Convergence in probability}

We start with the convergence in probability of the processes $X^{n}(g, h)$. We need the following structural assumption on $Y$ :

Hypothesis (H): We have

$$
Y_{t}=Y_{0}+\int_{0}^{t} a_{s} d s+\int_{0}^{t} \sigma_{s-} d W_{s}
$$

where $W$ is a standard $d^{\prime}$-dimensional BM, $a$ is predictable $\mathbf{R}^{d \text {-valued locally }}$ bounded, and $\sigma$ is $\mathcal{M}_{d, d^{\prime}}$-valued càdlàg.

Below $\rho_{\Sigma}$ denotes the normal law $\mathcal{N}\left(0, \Sigma \Sigma^{\star}\right)$, and $\rho_{\Sigma}(g)$ is the integral of $g$ w.r.t. $\rho_{\Sigma}$.

Theorem 2.1. Under $(H)$ and when the functions $g$ and $h$ are continuous with at most polynomial growth, we have

$$
X^{n}(g, h)_{t} \rightarrow X(g, h)_{t}:=\int_{0}^{t} \rho_{\sigma_{s}}(g) \rho_{\sigma_{s}}(h) d s,
$$

where the convergence is local uniform in time, and in probability.

If we apply this with the functions $g=f_{j, r}$ and $h=f_{k, s}$, we get a result of existence for the bipower variation processes. We denote by $\mu_{r}$ the $r$ th absolute moment of the law $\mathcal{N}(0,1)$. 
Theorem 2.2. Under (H), and if $r, s \geq 0$, we have

$$
V\left(Y^{j}, Y^{k} ; r, s\right)_{t}^{n} \rightarrow V\left(Y^{j}, Y^{k} ; r, s\right)_{t}:=\mu_{r} \mu_{s} \int_{0}^{t}\left|\sigma_{u}^{j j}\right|^{r}\left|\sigma_{u}^{k k}\right|^{s} d u
$$

where the convergence is local uniform in time, and in probability.

This result is essentially taken from [4]. The assumption $(\mathrm{H})$ could be weakened, of course, but probably not in any essential way. For instance the càdlàg hypothesis on $\sigma$ can be relaxed, but we need at least the functions $u \mapsto\left|\sigma_{u}^{j j}\right|^{r}$ to be Riemann-integrable, for all (or $\mathbf{P}$-almost all) $\omega$. The fact that the driving terms in (2.3) are $t$ and $W_{t}$ is closely related to the fact that the discretization in time has a constant step $1 / n$. If we replace (2.3) by

$$
Y_{t}=Y_{0}+\int_{0}^{t} a_{s} d A_{s}+\int_{0}^{t} \sigma_{s-} d M_{s}
$$

where $A$ is a continuous increasing process and $M$ a continuous martingale, then a result like (2.5) can hold only for discretization along increasing sequences of stopping times, related in some way to $A$ and to the quadratic variation of $M$. If further $Y$ is discontinuous, this type of result cannot possibly hold (with the normalizing factor $n^{\frac{r+s}{2}-1}$ ), as is easily seen when $Y$ is a simple discontinuous process like a Poisson process. As a matter of fact, this observation was the starting point of the papers [4] and [5] for introducing bipower variations, in order to discriminate between continuous and discontinuous processes.

Finally, we state the multipower variation result: the processes of (1.4) converge (under $(\mathrm{H})$ ) towards

$$
V\left(Y^{j_{1}}, \ldots, Y^{j_{N}} ; r_{1}, \ldots, r_{N}\right)_{t}=\mu_{r_{1}} \ldots \mu_{r_{N}} \int_{0}^{t}\left|\sigma_{u}^{j_{1} j_{1}}\right|^{r_{1}} \ldots\left|\sigma_{u}^{j_{N} j_{N}}\right|^{r_{N}} d u .
$$

\subsection{The central limit theorem}

For the CLT we need some additional structure on the volatility $\sigma$. A relatively simple assumption is then:

Hypothesis (H0): We have (H) with

$$
\sigma_{t}=\sigma_{0}+\int_{0}^{t} a_{s}^{\prime} d s+\int_{0}^{t} \sigma_{s-}^{\prime} d W_{s}+\int_{0}^{t} v_{s-} d Z_{s}
$$

where $Z$ is a $d^{\prime \prime}$-dimensional Lévy process on $\left(\Omega, \mathcal{F},\left(\mathcal{F}_{t}\right)_{t \geq 0}, \mathbf{P}\right)$, independent of $W$ (and possibly with a non-vanishing continuous martingale part). Furthermore the processes $\sigma^{\prime}$ and $v$, and $a$ of (2.7), are adapted càdlàg, with values in $\mathcal{M}_{d, d^{\prime}, d^{\prime}}$ and $\mathcal{M}_{d, d^{\prime}, d^{\prime \prime}}$ and $\mathcal{M}_{d, d^{\prime}}$ respectively, and $a^{\prime}$ is $\mathcal{M}_{d, d^{\prime}}$-valued, predictable and locally bounded. 
This assumption is in fact not general enough for applications. Quite often the natural ingredient in our model is the "square" $c=\sigma \sigma^{*}$ rather than $\sigma$ itself, and it is this $c$ which satisfies an equation like (2.7). In this case the "square-root" $\sigma$ of $c$ does not usually satisfy a similar equation. This is why we may replace (H0) by the following assumption:

Hypothesis (H1): We have $(\mathrm{H})$ with

$$
\begin{gathered}
\sigma_{t}=\sigma_{0}+\int_{0}^{t} a_{s}^{\prime} d s+\int_{0}^{t} \sigma_{s-}^{\prime} d W_{s}+\int_{0}^{t} v_{s-} d V_{s}+ \\
\int_{0}^{t} \int_{E} \varphi \circ w(s-, x)(\mu-\nu)(d s, d x)+\int_{0}^{t} \int_{E}(w-\varphi \circ w)(s-, x) \mu(d s, d x) .
\end{gathered}
$$

Here $a^{\prime}$ and $\sigma^{\prime}$ and $v$ are like in (H0); $V$ is a $d^{\prime \prime}$-dimensional Wiener process independent of $W$, with an arbitrary covariance structure; $\mu$ is a Poisson random measure on $(0, \infty) \times E$ independent of $W$ and $V$, with intensity measure $\nu(d t, d x)=d t F(d x)$ and $F$ is a $\sigma$-finite measure on the Polish space $(E, \mathcal{E})$; $\varphi$ is a continuous truncation function on $\mathbf{R}^{d d^{\prime}}$ (a function with compact support, which coincides with the identity map on a neigbourhood of 0 ); finally $w(\omega, s, x)$ is a map $\Omega \times[0, \infty) \times E \rightarrow \mathcal{M}_{d, d^{\prime}}$ which is $\mathcal{F}_{s} \otimes \mathcal{E}$-measurable in $(\omega, x)$ for all $s$ and càdlàg in $s$, and such that for some sequence $\left(S_{k}\right)$ of stopping times increasing to $+\infty$ we have:

$$
\sup _{\omega \in \Omega, s<S_{k}(\omega)}\|w(\omega, s, x)\| \leq \psi_{k}(x), \quad \text { where } \int_{E}\left(1 \bigwedge \psi_{k}(x)^{2}\right) F(d x)<\infty .
$$

This hypothesis looks complicated, but it is usually simple to check. The conditions on the coefficients imply in particular that all integrals in (2.8) are well defined. It is weaker than (H0): indeed if (H0) holds, we also have (H1) with $E=\mathbf{R}^{d^{\prime \prime}}$ and $V$ being the Wiener part of $Z$ if it exists, and $\mu$ being the random measure associated with the jumps of $Z$ (so $F$ is the Lévy measure of $Z$ ), and $w(\omega, t, x)=v_{t}(\omega) x$ (note that $v$ is the same in (2.7) and in (2.8); the processes $a^{\prime}$ in the two formulae are different, depending on the drift of $Z$ ).

We also sometimes need an additional assumption:

Hypothesis $\left(\mathbf{H}^{\prime}\right)$ : The process $\sigma \sigma^{\star}$ is everywhere invertible.

Set once more $c=\sigma \sigma^{*}$. If the processes $c$ and $c_{-}$are invertible, (H1) holds if and only if the process $c$ satisfies an equation like (2.8), with the same assumptions on the coefficients. This is not longer true if we replace (H1) and (2.8) by ( $\mathrm{H} 0)$ and (2.7).

As for the functions $g$ and $h$, we will suppose that their components satisfy one of the following assumptions, which we write for a real-valued function $f$ on $\mathbf{R}^{d}$; if $f$ is differentiable at $x$, we write $\nabla f(x)$ for the row matrix of its partial derivatives: 
Hypothesis (K): The function $f$ is even (that is, $f(-x)=f(x)$ for all $x \in \mathbf{R}^{d}$ ) and continuously differentiable, with partial derivatives having at most polynomial growth.

Hypothesis ( $\left.\mathbf{K}^{\prime}\right)$ : The function $f$ is even and continuously differentiable on the complement $B^{c}$ of a closed subset $B \subset \mathbf{R}^{d}$ and satisfies

$$
\|y\| \leq 1 \Rightarrow|f(x+y)-f(x)| \leq C\left(1+\|x\|^{p}\right)\|y\|^{r}
$$

for some constants $C>0, p \geq 0$ and $r \in(0,1]$. Moreover:

a) If $r=1$ then $B$ has Lebesgue measure 0 .

b) If $r<1$ then $B$ satisfies

for any positive definite $d \times d$ matrix $C$ and any $\mathcal{N}(0, C)$-random vector $U$ the distance $d(U, B)$ from $U$ to $B$ has a density $\psi_{C}$ on $\mathbf{R}_{+}$, such that $\sup _{x \in \mathbf{R}_{+},\|C\|+\left\|C^{-1}\right\| \leq A} \psi_{C}(x)<\infty$ for all $A<\infty$,

and we have

$$
x \in B^{c},\|y\| \leq 1 \bigwedge \frac{d(x, B)}{2} \Rightarrow\left\{\begin{array}{l}
\|\nabla f(x)\| \leq \frac{C\left(1+\|x\|^{p}\right)}{d(x, B)^{1-r}}, \\
\|\nabla f(x+y)-\nabla f(x)\| \leq \frac{C\left(1+\|x\|^{p}\right)\|y\|}{d(x, B)^{2-r}} .
\end{array}\right.
$$

The additional requirements when $r<1$ above are not "optimal", but they accomodate the case where $f$ equals $f_{j, r}$, as defined in (2.2): this function satisfies (K) when $r>1$, and (K') when $r \in(0,1]$ (with the same $r$ of course). When $B$ is a finite union of hyperplanes it satisfies (2.11). Also, observe that (K) implies (K') with $r=1$ and $B=\emptyset$. For the concept of "stable convergence in law", introduced by Renyi in [11], we refer to [9] for example; it is a kind of convergence which is a bit stronger than the ordinary convergence in law.

Theorem 2.3. Under (H1) (or (HO)) and either one the following assumptions:

(i) all components of $g$ and $h$ satisfy $(K)$,

(ii) $\left(H^{\prime}\right)$ holds, and all components of $g$ and $h$ satisfy ( $\left.K^{\prime}\right)$,

the processes $\sqrt{n}\left(X^{n}(g, h)-X(g, h)\right)$ converge stably in law towards the limiting process $U(g, h)$ given componentwise by

$$
U(g, h)_{t}^{j k}=\sum_{j^{\prime}=1}^{d_{1}} \sum_{k^{\prime}=1}^{d_{3}} \int_{0}^{t} \alpha\left(\sigma_{s}, g, h\right)^{j k, j^{\prime} k^{\prime}} d W_{s}^{\prime j^{\prime} k^{\prime}}
$$

where 


$$
\left.\begin{array}{r}
\sum_{l=1}^{d_{1}} \sum_{m=1}^{d_{3}} \alpha(\Sigma, g, h)^{j k, l m} \alpha(\Sigma, g, h)^{j^{\prime} k^{\prime}, l m}=A(\Sigma, g, h)^{j k, j^{\prime} k^{\prime}} \\
\text { and } A(\Sigma, g, h)^{j k, j^{\prime} k^{\prime}}=\sum_{l, l^{\prime}=1}^{d_{2}}\left(\rho_{\Sigma}\left(g^{j l} g^{j^{\prime} l^{\prime}}\right) \rho_{\Sigma}\left(h^{l k} h^{l^{\prime} k^{\prime}}\right)\right. \\
+\rho_{\Sigma}\left(g^{j l}\right) \rho_{\Sigma}\left(h^{l^{\prime} k^{\prime}}\right) \rho_{\Sigma}\left(g^{j^{\prime} l^{\prime}} h^{l k}\right)+\rho_{\Sigma}\left(g^{j^{\prime} l^{\prime}}\right) \rho_{\Sigma}\left(h^{l k}\right) \rho_{\Sigma}\left(g^{j l} h^{l^{\prime} k^{\prime}}\right) \\
\left.-3 \rho_{\Sigma}\left(g^{j l}\right) \rho_{\Sigma}\left(g^{j^{\prime} l^{\prime}}\right) \rho_{\Sigma}\left(h^{l k}\right) \rho_{\Sigma}\left(h^{l^{\prime} k^{\prime}}\right)\right),
\end{array}\right\}
$$

and $W^{\prime}$ is a $d_{1} d_{3}$-dimensional Wiener process which is defined on an extension of the space $\left(\Omega, \mathcal{F},\left(\mathcal{F}_{t}\right)_{t \geq 0}, \mathbf{P}\right)$ and is independent of the $\sigma$-field $\mathcal{F}$.

The first formula in (2.14) means that $\alpha$ is a square-root of the $d_{1} d_{3} \times d_{1} d_{3}-$ matrix $A$, which is symmetric semi-definite positive. Observe that the right sides of (2.4) and (2.13) always make sense, due to the fact that $t \mapsto \sigma_{t}$ is càdlàg and thus with all powers locally integrable w.r.t. Lebesgue measure.

Under $(\mathrm{H})$ and if both $g$ and $h$ are even and continuous, the processes

$$
\begin{aligned}
U^{n}(f, g)_{t}=\frac{1}{\sqrt{n}} & \sum_{i=1}^{[n t]}\left(g\left(\sqrt{n} \Delta_{i}^{n} Y\right) h\left(\sqrt{n} \Delta_{i+1}^{n} Y\right)\right. \\
& \left.-\mathbf{E}\left(g\left(\sqrt{n} \Delta_{i}^{n} Y\right) h\left(\sqrt{n} \Delta_{i+1}^{n} Y\right) \mid \mathcal{F}_{\frac{i-1}{n}}\right)\right)
\end{aligned}
$$

still converge stably in law to $U(g, h)$ provided $a$ and $\sigma$ have some integrability properties in connection with the growth rate of $g$ and $h$ (so that the conditional expectations above are meaningful): see Theorem 5.1 below for a version of this when $a$ and $\sigma$ are bounded. But such a CLT is probably of little practical use.

Remarks: For simplicity we state the remarks when all processes are 1dimensional and when $h(x)=1$.

1. When $g$ is not even we still have a limiting process which is the process $U(g, 1)$ plus a process which has a drift and an integral term w.r.t. $W$ : for example if $g(x)=x$, then $X(g, 1)=0$ and of course $\sqrt{n} X^{n}(g, h)_{t}=$ $Y_{[n t] / n}$, so the limit is $Y$ itself (in this case $U(g, 1)=0$ ). For more details, see $[8]$.

2. In view of the result on (2.15), when $h=1$ the CLT is essentially equivalent to the convergence of

$$
\frac{1}{\sqrt{n}} \sum_{i=1}^{[n t]}\left(\mathbf{E}\left(g\left(\sqrt{n} \Delta_{i}^{n} Y\right) \mid \mathcal{F}_{\frac{i-1}{n}}\right)-n \int_{\frac{i-1}{n}}^{\frac{i}{n}} \rho_{\sigma_{u}}(g) d u\right)
$$

to 0 (locally uniform in $t$ ). This in turn is implied by the convergence to 0 of the following two processes: 


$$
\begin{gathered}
\frac{1}{\sqrt{n}} \sum_{i=1}^{[n t]}\left(\mathbf{E}\left(g\left(\sqrt{n} \Delta_{i}^{n} Y\right) \mid \mathcal{F}_{\frac{i-1}{n}}\right)-\mathbf{E}\left(g\left(\sqrt{n} \sigma_{\frac{i-1}{n}} \Delta_{i}^{n} W\right) \mid \mathcal{F}_{\frac{i-1}{n}}\right)\right), \\
\frac{1}{\sqrt{n}} \sum_{i=1}^{[n t]}\left(\rho_{\frac{\sigma_{-1}}{n}}(g)-n \int_{\frac{i-1}{n}}^{\frac{i}{n}} \rho_{\sigma_{u}}(g) d u\right) .
\end{gathered}
$$

3. For (2.17) we need some smoothness of $\sigma$ : e.g. $u \mapsto \sigma_{u}$ is Hölder with some index $>1 / 2$. Hypothesis (H1) is of this kind (although $\sigma$ can have jumps, (2.8) sort of implies that it is "Hölder" of order $1 / 2$ and further some compensation arises).

4. The differentiability of $g$ is in fact used for the convergence of (2.16). Another natural idea would be to compare the transition densities of $Y$ and $W$ for small times, provided of course the former ones exist: that allows to get the results for functions $g$ and $h$ which are only Borelmeasurable, in Theorem 2.3 and in Theorem 2.1 as well, but it necessitates quite stringent assumptions on $Y$ (like a Markov structure, and nondegeneracy).

\subsection{Applications to bipower variations}

Let us now explain how the general CLT above writes for bipower variations. The most general form is given below, but for simplicity we first consider the 1-dimensional case for $Y$, with a single bipower process.

Theorem 2.4. Let $r, s \geq 0$ and assume that $d=d^{\prime}=1$. Assume (H1) and also that either $r, s \in\{0\} \cup(1, \infty)$ or $\left(H^{\prime}\right)$ holds. Then the processes $\left(\sqrt{n}\left(V(Y ; r, s)^{n}-V(Y ; r, s)\right)\right)$ converge stably in law to a process $U(r, s)$ of the form

$$
U(r, s)_{t}=\sqrt{\mu_{2 r} \mu_{2 s}+2 \mu_{r} \mu_{s} \mu_{r+s}-3 \mu_{r}^{2} \mu_{s}^{2}} \int_{0}^{t}\left|\sigma_{u}\right|^{r+s} d W_{u}^{\prime},
$$

where $W^{\prime}$ is a Wiener process which is defined on an extension of the space $\left(\Omega, \mathcal{F},\left(\mathcal{F}_{t}\right)_{t \geq 0}, \mathbf{P}\right)$ and is independent of the $\sigma$-field $\mathcal{F}$.

For the general case we consider simultaneously all cross-bipower variations for any finite family of indices. We need some more notation: we denote by $\mu(\Sigma ; r, s ; j, k)$ the expected value of $\left|U_{j}\right|^{r}\left|U_{k}\right|^{s}$ when $U=\left(U_{j}\right)_{1 \leq j \leq d}$ is an $\mathcal{N}\left(0, \Sigma \Sigma^{*}\right)$-distributed random variable, and also by $\mu(\Sigma ; r ; j)$ the expected value of $\left|U_{j}\right|^{r}$ (so $\mu(\Sigma ; r ; j)=\mu(\Sigma ; r, 0 ; j, k)$ for any $k$, and $\mu(\Sigma ; r ; j)=$ $\left|C^{j j}\right|^{r / 2} \mu_{r}$, where $\left.C=\Sigma \Sigma^{*}\right)$.

Theorem 2.5. Let $\left(r_{l}, s_{l}\right)$ be a family of nonnegative reals. Under (H1) and either one of the following assumptions:

(i) $r_{l}, s_{l} \in\{0\} \cup(1, \infty)$,

(ii) $\left(H^{\prime}\right)$ and $r_{l}, s_{l} \in[0, \infty)$,

the $L \times d \times d$-dimensional processes 
$\left(\sqrt{n}\left(V\left(Y^{j}, Y^{k} ; r_{l}, s_{l}\right)^{n}-V\left(Y^{j}, Y^{k} ; r_{l}, s_{l}\right)\right): 1 \leq l \leq L, 1 \leq j, k \leq d\right)$

converge stably in law to a process $\left(U\left(r_{l}, s_{l}, j, k\right): 1 \leq l \leq L, 1 \leq j, k \leq d\right)$ having the form

$$
U\left(r_{l}, s_{l}, j, k\right)_{t}=\sum_{l^{\prime}=1}^{L} \sum_{j^{\prime}=1}^{d} \sum_{k^{\prime}=1}^{d} \int_{0}^{t} \alpha\left(\sigma_{u}\right)^{l j k, l^{\prime} j^{\prime} k^{\prime}} d W_{u}^{\prime l^{\prime} j^{\prime} k^{\prime}},
$$

where

$$
\left.\begin{array}{rl}
\sum_{l^{\prime \prime}=1}^{L} \sum_{j^{\prime \prime}=1}^{d} \sum_{k^{\prime \prime}=1}^{d} & \alpha(\Sigma)^{l j k, l^{\prime \prime} j^{\prime \prime} k^{\prime \prime}} \alpha(\Sigma)^{l^{\prime} j^{\prime} k^{\prime}, l^{\prime \prime} j^{\prime \prime} k^{\prime \prime}}=A^{l j k, l^{\prime} j^{\prime} k} \\
\text { and } A(\Sigma)^{l j k, l^{\prime} j^{\prime} k^{\prime}} & =\mu\left(\Sigma ; r_{l}, r_{l^{\prime}} ; j, j^{\prime}\right) \mu\left(\Sigma ; s_{l}, s_{l^{\prime}} ; k, k^{\prime}\right) \\
& +\mu\left(\Sigma ; r_{l} ; j\right) \mu\left(\Sigma ; s_{l^{\prime}} ; k^{\prime}\right) \mu\left(\Sigma ; r_{l^{\prime}}, s_{l} ; j^{\prime}, k\right) \\
& +\mu\left(\Sigma ; r_{l^{\prime}} ; j^{\prime}\right) \mu\left(\Sigma ; s_{l} ; k\right) \mu\left(\Sigma ; r_{l}, s_{l^{\prime}} ; j, k^{\prime}\right) \\
& -3 \mu\left(\Sigma ; r_{l} ; j\right) \mu\left(\Sigma ; r_{l^{\prime}} ; j^{\prime}\right) \mu\left(\Sigma ; s_{l} ; k\right) \mu\left(\Sigma ; s_{l^{\prime}} ; k^{\prime}\right)
\end{array}\right\}
$$

and where $W^{\prime}$ is an $L \times d \times d$-dimensional Wiener process which is defined on an extension of $\left(\Omega, \mathcal{F},\left(\mathcal{F}_{t}\right)_{t \geq 0}, \mathbf{P}\right)$ and is independent of the $\sigma$-field $\mathcal{F}$.

This result readily follows from Theorem 2.3 , upon taking $d_{1}=L d, d_{2}=L$, $d_{3}=d, g(x)^{l j, l^{\prime}}=\left|x^{j}\right|^{r_{l}} \varepsilon_{l l^{\prime}}\left(\varepsilon_{l l^{\prime}}\right.$ is the Kronecker symbol) and $h(x)^{l, j}=\left|x^{j}\right|^{s_{l}}$. Apart from Theorem 2.4, several particular cases are worth being mentioned (recall that $c=\sigma \sigma^{*}$ ):

1. If $j=k$ then $\sqrt{n}\left(V\left(Y^{j} ; r, s\right)^{n}-V\left(Y^{j} ; r, s\right)\right)$ stably converges to

$$
\sqrt{\mu_{2 r} \mu_{2 s}+2 \mu_{r} \mu_{s} \mu_{r+s}-3 \mu_{r}^{2} \mu_{s}^{2}} \int_{0}^{t}\left|c_{u}^{j j}\right|^{\frac{r+s}{2}} d W_{u}^{\prime} .
$$

This is also, of course, a consequence of Theorem 2.4 .

2. The bivariate processes with components $\sqrt{n}\left(V\left(Y^{j} ; r, 0\right)^{n}-V\left(Y^{j} ; r, 0\right)\right)$ and $\sqrt{n}\left(V\left(Y^{k} ; 0, s\right)^{n}-V\left(Y^{k} ; 0, s\right)\right)$ stably converge to a continuous martingale with (matrix-valued) bracket $C$ given by

$$
\left.\begin{array}{l}
C_{t}^{11}=\left(\mu_{2 r}-\mu_{r}^{2}\right) \int_{0}^{t}\left|c_{u}^{j j}\right|^{r} d u \\
C_{t}^{12}=\int_{0}^{t}\left(\mu\left(\sigma_{u} ; r, s ; j, k\right)-\mu_{r} \mu_{s}\left|c_{u}^{j j}\right|^{r / 2}\left|c_{u}^{k k}\right|^{s / 2}\right) d u \\
C_{t}^{22}=\left(\mu_{2 s}-\mu_{s}^{2}\right) \int_{0}^{t}\left|c_{u}^{j j}\right|^{s} d u
\end{array}\right\} .
$$

The same is true for the processes with components $\sqrt{n}\left(V\left(Y^{j} ; r, 0\right)^{n}-\right.$ $\left.V\left(Y^{j} ; r, 0\right)\right)$ and $\sqrt{n}\left(V\left(Y^{k} ; s, 0\right)^{n}-V\left(Y^{k} ; s, 0\right)\right)$. When $j=k$ we get $C_{t}^{12}=\left(\mu_{r+s}-\mu_{r} \mu_{s}\right) \int_{0}^{t}\left|c_{u}^{j j}\right|^{\frac{r+s}{2}} d u$. 
Finally we state the multipower variation result, in the 1-dimensional case only for simplicity. We consider the processes of (1.4) and (2.6), which are written $V\left(Y ; r_{1}, \ldots, r_{N}\right)^{n}$ and $V\left(Y ; r_{1}, \ldots, r_{N}\right)$ here. For any choice of $r_{l} \geq 0$, and under (H1) and also under ( $\left.\mathrm{H}^{\prime}\right)$ if any of the $r_{l}$ is in the set $(0,1]$, the processes $\sqrt{n}\left(V\left(Y ; r_{1}, \ldots, r_{N}\right)^{n}-V\left(Y ; r_{1}, \ldots, r_{N}\right)\right)$ converge stably towards a limiting process of the form

$$
U\left(r_{1}, \ldots, r_{N}\right)_{t}=\sqrt{A} \int_{0}^{t}\left|\sigma_{u}\right|^{r_{1}+\ldots+r_{N}} d W_{u}^{\prime},
$$

where $W^{\prime}$ is a Wiener process independent of the $\sigma$-field $\mathcal{F}$, and where

$$
A=\prod_{l=1}^{N} \mu_{2 r_{l}}-(2 N-1) \prod_{l=1}^{N} \mu_{r_{l}}^{2}+2 \sum_{k=1}^{N-1} \prod_{l=1}^{k} \mu_{r_{l}} \prod_{l=N-k+1}^{N} \mu_{r_{l}} \prod_{l=1}^{N-k} \mu_{r_{l}+r_{l+k}} .
$$

\subsection{Outline of the proof}

The remainder of this paper is devoted to proving Theorems 2.1 and 2.3:

1. In Section 3 we replace the "local" assumptions $(\mathrm{H}),(\mathrm{H} 1)$ and $(\mathrm{H}$ ') by "global" ones called (SH), (SH1) and (SH'): these stronger assumptions are likely to be satisfied in many practical applications, and the "localization techniques" using stopping times are standard: so the reader can very well skip most of that section and read only the assumptions and (3.6).

2. The idea of the proof is simple enough. First, replace the increments $\Delta_{i}^{n} Y$ of the process (2.3) by $\sigma_{(i-1) / n} \Delta_{i}^{n} W$ : then the CLT is a simple consequence of the convergence of triangular arrays of martingale differences, and the convergence in probability follows from the CLT: this is basically the content of Section 4. In Section 5 we prove the CLT for the processes of (2.15): this easily follows from Section 4 . Hence proving Theorems 2.1 and 2.3 amounts to control of the differences $X^{n}(g, h)-U^{n}(g, h)$ or $\sqrt{n}\left(X^{n}(g, h)-U^{n}(g, h)\right)$ : for Theorem 2.1 this is simple, see Section 6 . For Theorem 2.3 it is done in Section 8: we have to split the above differences into a large number of terms, which are estimated separately. So we gather the necessary (very cumbersome) notation and technical estimates in Section 7.

\section{Some stronger assumptions}

Under $(\mathrm{H})$ we have a sequence $T_{k}$ of stopping times increasing to $+\infty$ and constants $C_{k}$ such that

$$
s \leq T_{k} \quad \Longrightarrow \quad\left|a_{s}\right|+\left|\sigma_{s-}\right| \leq C_{k} .
$$


Set $a_{s}^{(k)}=a_{s \wedge T_{k}}$, and $\sigma_{s}^{(k)}=\sigma_{s}$ if $s<T_{k}$ and $\sigma_{s}^{(k)}=\sigma_{T_{k}-}$ if $s \geq T_{k}$. We associate $Y^{(k)}$ with $a^{(k)}$ and $\sigma^{(k)}$ by $(2.3)$, and $X^{n,(k)}(g, h)$ with $Y^{(k)}$ by $(2.1)$, and similarly $X^{(k)}(g, h)$ and $U^{(k)}(g, h)$ with $\sigma^{(k)}$ by (2.4) and (2.13) (and the same process $W^{\prime}$ for all $k$ ).

Suppose that we have proved Theorem 2.1 for $X^{n,(k)}(g, h)$, for each $k$. Observing that $X^{n,(k)}(g, h)_{t}=X^{n}(g, h)_{t}$ and $X^{(k)}(g, h)_{t}=X(g, h)_{t}$ and $U^{(k)}(g, h)_{t}=U(g, h)_{t}$ for all $t<T_{k}$, and since $T_{k}$ increases to $\infty$ as $k \rightarrow \infty$, it is obvious that the result of Theorem 2.1 also holds for $X^{n}(g, h)$. So, instead of $(\mathrm{H})$, it is no restriction for proving Theorem 2.1 to assume the following stronger hypothesis:

Hypothesis (SH): We have (H), and further the processes $a$ and $\sigma$ are bounded by a constant.

Now we proceed to strenghten (H1) in a similar manner. Assume (H1) and recall the sequence $\left(S_{k}\right)$ in $(2.9)$ : it is no restriction to assume in addition that $S_{k} \leq k$. Set for $k, l \geq 1$ :

$$
E_{k, l}=\left\{x \in E: \psi_{k}(x)>l\right\}, \quad R_{k, l}=\inf \left(t: \mu\left((0, t] \times E_{k, l}\right) \geq 1\right) .
$$

Then we have

$$
\mathbf{P}\left(R_{k, l} \leq S_{k}\right) \leq \mathbf{E}\left(\mu\left(\left(0, S_{k}\right] \times E_{k, l}\right)\right)=F\left(E_{k, l}\right) \mathbf{E}\left(S_{k}\right) \leq k F\left(E_{k, l}\right) .
$$

In view of (2.9) we have $\lim _{l \rightarrow \infty} F\left(E_{k, l}\right)=0$. Hence we find $l_{k}$ such that $\mathbf{P}\left(R_{k, l_{k}}<S_{k}\right) \leq 2^{-k}$, and obviously the sequence of stopping times $S_{k}^{\prime}=$ $S_{k} \wedge R_{k, l_{k}}$ has $\sup _{k} S_{k}^{\prime}=\infty$ a.s.

Next, just as above, we find a sequence $S_{k}^{\prime \prime}$ of stopping times increasing to $+\infty$ and constants $C_{k}$ such that

$$
s \leq S_{k}^{\prime \prime} \quad \Longrightarrow \quad\left\|a_{s}\right\|+\left\|\sigma_{s-}\right\|+\left\|a_{s}^{\prime}\right\|+\left\|\sigma_{s-}^{\prime}\right\|+\left\|v_{s-}\right\| \leq C_{k} .
$$

Then if $T_{k}=S_{k}^{\prime} \wedge S_{k}^{\prime \prime}$, we still have $\sup _{k} T_{k}=\infty$ a.s., and further

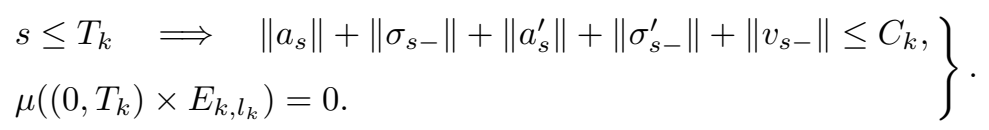

Set

$$
\begin{aligned}
& a_{s}^{\prime(k)}=\left\{\begin{array}{l}
a_{s}^{\prime} \text { if } s \leq T_{k} \\
0 \text { if } s>T_{k}
\end{array}\right. \\
& \left(a_{s}^{(k)}, \sigma_{s}^{\prime(k)}, v_{s}^{(k)}, w^{(k)}(s, x)\right)= \begin{cases}\left(a_{s}, \sigma_{s}^{\prime}, v_{s}, w(s, x)\right) & \text { if } s<T_{k} \\
(0,0,0,0) & \text { if } s \geq T_{k},\end{cases} \\
& \mu^{(k)}(d s, d x)=\mu(d s, d x) 1_{E_{k, l_{k}}^{c}}(x),
\end{aligned}
$$




$$
\nu^{(k)}(d s, d x)=d s \otimes F_{k}(d x), \quad \text { where } F_{k}(d x)=F(d x) 1_{E_{k, l_{k}}^{c}}(x) .
$$

Then $\mu^{(k)}$ is a new Poisson measure, still independent of $W$ and $V$, with compensator $\nu^{(k)}$, and $\psi_{k}$ is square-integrable w.r.t. $F_{k}$. We then put

$$
\begin{aligned}
\sigma_{t}^{(k)}= & \sigma_{0}+\int_{0}^{t} a_{s}^{\prime(k)} d s+\int_{0}^{t} \sigma_{s-}^{\prime(k)} d W_{s}+\int_{0}^{t} v_{s-}^{(k)} d V_{s} \\
& +\int_{0}^{t} \int_{E} \varphi \circ w^{(k)}(s-, x)\left(\mu^{(k)}-\nu^{(k)}\right)(d s, d x) \\
& +\int_{0}^{t} \int_{E}\left(w^{(k)}-\varphi \circ w^{(k)}\right)(s-, x) \mu^{(k)}(d s, d x) \\
= & \sigma_{0}+\int_{0}^{t}\left(a_{s}^{\prime(k)}+\alpha_{s}^{(k)}\right) d s+\int_{0}^{t} \sigma_{s-}^{\prime(k)} d W_{s}+\int_{0}^{t} v_{s-}^{(k)} d V_{s} \\
& +\int_{0}^{t} \int_{E} w^{(k)}(s-, x)\left(\mu^{(k)}-\nu^{(k)}\right)(d s, d x),
\end{aligned}
$$

provided $\alpha_{s}^{(k)}=\int_{E}\left(w^{(k)}-\varphi \circ w^{(k)}\right)(s-, x) F_{k}(d x)$. Then $\sigma_{s}^{(k)}=\sigma_{s}$ when $s<T_{k}$ and $\left\|\alpha_{s}^{(k)}\right\| \leq C_{k}^{\prime}$ for all $s$, for some constant $C_{k}^{\prime}$.

We associate $Y^{(k)}$ with $a^{(k)}$ and $\sigma^{(k)}$ by $(2.3)$, and $X^{n,(k)}(g, h)$ with $Y^{(k)}$ by $(2.1)$, and similarly $X^{(k)}(g, h)$ and $U^{(k)}(g, h)$ with $\sigma^{(k)}$ by (2.4) and (2.13) (and the same process $W^{\prime}$ for all $k$ ). We clearly have $X^{n,(k)}(g, h)_{t}=X^{n}(g, h)_{t}$ and $X^{(k)}(g, h)_{t}=X(g, h)_{t}$ and $U^{(k)}(g, h)_{t}=U(g, h)_{t}$ for all $t<T_{k}$.

Hence, exactly as for $(\mathrm{H})$, for proving Theorem 2.3 it is no restriction to replace (H1) by the following stronger assumption (recall (3.3)):

Hypothesis (SH1): We have (SH) with

$$
\sigma_{t}=\sigma_{0}+\int_{0}^{t} a_{s}^{\prime} d s+\int_{0}^{t} \sigma_{s-}^{\prime} d W_{s}+\int_{0}^{t} v_{s-} d V_{s}+\int_{0}^{t} \int_{E} w(s-, x)(\mu-\nu)(d s, d x)
$$

with $V, \mu$ and $\nu$ as in (H1), and $a^{\prime}, \sigma^{\prime}, v$ and $a$ are like in (H0) and uniformly bounded. Finally $w$ is like in (H1), with further

$$
\sup _{\omega \in \Omega, s \geq 0}\|w(\omega, s, x)\| \leq \psi(x), \quad \text { where } \int_{E} \psi(x)^{2} F(d x)<\infty, \quad \psi(x) \leq C .
$$

In a similar way, under $\left(\mathrm{H}^{\prime}\right)$ we find a sequence $T_{k}$ of stopping times satisfying (3.1) and also $\left\|\left(\sigma_{s} \sigma_{s}^{\star}\right)^{-1}\right\| \leq C_{k}$ if $s<T_{k}$. So the same argument as above allows to replace (H') in Theorem 2.3 by

Hypothesis ( $\left.\mathbf{S H}^{\prime}\right)$ : We have $\left(\mathrm{H}^{\prime}\right)$ and further the process $\left(\sigma \sigma^{\star}\right)^{-1}$ is bounded.

Finally, let us denote by $\mathcal{M}^{\prime}$ the closure of the set $\left\{\sigma_{u}(\omega): \omega \in \Omega, u \geq 0\right\}$ in $\mathcal{M}_{d, d^{\prime}}$. Then there is a constant $A_{0}$ such that: 


$$
\left.\begin{array}{llll}
\text { under (SH) we have } \quad \Sigma \in \mathcal{M}^{\prime} & \Rightarrow & \|\Sigma\| \leq A_{0} \\
\text { under (SH') we have } \quad \Sigma \in \mathcal{M}^{\prime} & \Rightarrow & \left\|\left(\Sigma \Sigma^{\star}\right)^{-1}\right\| \leq A_{0} .
\end{array}\right\}
$$

In view of the previous results, we can and will assume in the sequel either (SH), or (SH1), and sometimes (SH').

Let us also fix some conventions. We write $V^{n} \stackrel{\mathbf{P}}{\longrightarrow} V$ for a sequence $\left(V^{n}\right)$ of processes and a continuous process $V$ when $\sup _{s \leq t}\left\|V_{s}^{n}-V_{s}\right\|$ goes to 0 in probability for all $t>0$. When $V^{n}$ takes the form $V_{t}^{n}=\sum_{i=1}^{[n t]} \zeta_{i}^{n}$ for an array of variables $\left(\zeta_{i}^{n}\right)$, and when $V^{n} \stackrel{\mathbf{P}}{\longrightarrow} 0$, we say that this array is $A N$, for Asymptotically Negligible.

The constants occuring here and there may depend on the constants in (SH) or (SH1) and on the functions $g$ and $h$ and are all denoted by $C$ and change from line to line; if they depend on another external parameter $p$, we write them $C_{p}$.

\section{A first simplified problem}

In this section we prove the CLT in a slightly different setting: in some sense, we pretend that at stage $n, \sigma$ is constant over the interval $[(i-1) / n, i / n)$. More precisely, we introduce the following $\mathbf{R}^{d}$-valued random variables:

$$
\beta_{i}^{n}=\sqrt{n} \sigma_{\frac{i-1}{n}} \Delta_{i}^{n} W, \quad \beta_{i}^{\prime n}=\sqrt{n} \sigma_{\frac{i-1}{n}} \Delta_{i+1}^{n} W,
$$

and we write $\rho_{i}^{n}=\rho_{\sigma_{i / n}}$. To begin with, we consider an $\mathcal{M}_{d_{1}, d_{2}}$-valued adapted càdlàg and bounded process $\delta$ and an $\mathcal{M}_{d_{2}, d_{3}}$-valued function $f$ on $\mathbf{R}^{d}$. Then we introduce the $\mathcal{M}_{d_{1}, d_{3}}$-valued process (recall (4.1)):

$$
U_{t}^{n}=\frac{1}{\sqrt{n}} \sum_{i=1}^{[n t]} \delta_{\frac{i-1}{n}}\left(f\left(\beta_{i}^{n}\right)-\rho_{i-1}^{n}(f)\right)
$$

In a similar way, for $g$ and $h$ like in (2.1), we set

$$
U_{t}^{\prime n}=\frac{1}{\sqrt{n}} \sum_{i=1}^{[n t]}\left(g\left(\beta_{i}^{n}\right) h\left(\beta_{i}^{\prime n}\right)-\rho_{i-1}^{n}(g) \rho_{i-1}^{n}(h)\right) .
$$

Our aim in this section is then to prove the following two CLT's:

Proposition 4.1. Under $(S H)$, if $f$ is at most of polynomial growth, the sequence of processes $U^{n}$ in (4.2) is C-tight. If further $f$ is even, then it converges stably in law to the process $U$ defined componentwise by 


$$
U_{t}^{j k}=\sum_{j^{\prime}=1}^{d_{1}} \sum_{k^{\prime}=1}^{d_{3}} \int_{0}^{t} \delta_{u}^{j k k, j^{\prime} k^{\prime}} d W_{u}^{\prime j^{\prime} k^{\prime}}
$$

where

$$
\sum_{l=1}^{d_{1}} \sum_{m=1}^{d_{3}} \delta_{u}^{\prime j k, l m} \delta_{u}^{j^{\prime} k^{\prime}, l m}=\sum_{l, l^{\prime}=1}^{d_{2}}\left(\rho_{\sigma_{u}}\left(f^{l k} f^{l^{\prime} k^{\prime}}\right)-\rho_{\sigma_{u}}\left(f^{l k}\right) \rho_{\sigma_{u}}\left(f^{l^{\prime} k^{\prime}}\right)\right) \delta_{u}^{j l} \delta_{u}^{j^{\prime} l^{\prime}}
$$

and $W^{\prime}$ is a $d_{1} d_{3}$-dimensional Wiener process defined on an extension of $\left(\Omega, \mathcal{F},\left(\mathcal{F}_{t}\right)_{t \geq 0}, \mathbf{P}\right)$ and which is independent of the $\sigma$-field $\mathcal{F}$.

Proposition 4.2. Under (SH) and if $g$ and $h$ are continuous with at most polynomial growth, the sequence of processes $U^{\prime n}$ is $C$-tight. If further $g$ and $h$ are even, then it converges stably in law to the process $U(g, h)$ described in (2.13).

Before proceeding to the proofs, let us mention the following estimates, which are obvious under $(\mathrm{SH})$ :

$$
\mathbf{E}\left(\left\|\beta_{i}^{n}\right\|^{q}\right)+\mathbf{E}\left(\left\|\beta_{i}^{\prime n}\right\|^{q}\right) \leq C_{q}
$$

Next, saying that $f$ is of at most polynomial growth means that for some constants $C>0$ and $p$ (we can always choose $p \geq 2$ ),

$$
x \in \mathbf{R}^{d} \Rightarrow|f(x)| \leq C\left(1+\|x\|^{p}\right) .
$$

Observe also that Propositions 4.1 and 4.2 imply respectively

$$
\begin{gathered}
\frac{1}{n} \sum_{i=1}^{[n t]} \delta_{\frac{i-1}{n}} f\left(\beta_{i}^{n}\right) \stackrel{\mathbf{P}}{\longrightarrow} \int_{0}^{t} \delta_{u} \rho_{\sigma_{u}}(f) d u, \\
\frac{1}{n} \sum_{i=1}^{[n t]} g\left(\beta_{i}^{n}\right) h\left(\beta_{i}^{\prime n}\right) \stackrel{\mathbf{P}}{\longrightarrow} \int_{0}^{t} \rho_{\sigma_{u}}(g) \rho_{\sigma_{u}}(h) d u .
\end{gathered}
$$

Proof of Proposition 4.1. We have $U_{t}^{n}=\sum_{i=1}^{[n t]} \zeta_{i}^{n}$, where $\zeta_{i}^{n}=$ $\delta_{\frac{i-1}{n}}\left(f\left(\beta_{i}^{n}\right)-\rho_{i-1}^{n}(f)\right) / \sqrt{n}$. Recalling (4.6) and (4.7), we trivially have

$$
\begin{gathered}
\mathbf{E}\left(\zeta_{i}^{n} \mid \mathcal{F}_{\frac{i-1}{n}}\right)=0, \quad \mathbf{E}\left(\left\|\zeta_{i}^{n}\right\|^{4} \mid \mathcal{F}_{\frac{i-1}{n}}\right) \leq \frac{C}{n^{2}}, \\
\mathbf{E}\left(\zeta_{i}^{n, j k} \zeta_{i}^{n, j^{\prime} k^{\prime}} \mid \mathcal{F}_{\frac{i-1}{n}}\right)=\frac{1}{n} \Delta_{\frac{i-1}{n}}^{j k, j^{\prime} k^{\prime}}
\end{gathered}
$$

where $\Delta_{u}^{j k, j^{\prime} k^{\prime}}$ is the right side of (4.5). Moreover since $\sigma$ is càdlàg we deduce from (4.7) that $s \mapsto \rho_{\sigma_{s}}(f)$ also is càdlàg. Thus by the Riemann integrability we get 


$$
\sum_{i=1}^{[n t]} \mathbf{E}\left(\zeta_{i}^{n, j k} \zeta_{i}^{n, j^{\prime} k^{\prime}} \mid \mathcal{F}_{\frac{i-1}{n}}\right) \rightarrow \int_{0}^{t} \Delta_{u}^{j k, j^{\prime} k^{\prime}} d u
$$

Then (4.10) and (4.11) are enough to imply the tightness of the sequence $\left(U^{n}\right)$.

Now, assume further that $f$ is even. Since the variables $\Delta_{i}^{n} W$ and $-\Delta_{i}^{n} W$ have the same law, conditionally on $\mathcal{F}_{(i-1) / n}$, we get

$$
\mathbf{E}\left(\zeta_{i}^{n, j k} \Delta_{i}^{n} W^{l} \mid \mathcal{F}_{\frac{i-1}{n}}\right)=\sum_{m=1}^{d_{2}} \delta_{\frac{i-1}{n}}^{j m} \mathbf{E}\left(\Delta_{i}^{n} W^{l} f\left(\sqrt{n} \sigma_{\frac{i-1}{n}} \Delta_{i}^{n} W\right)^{m k} \mid \mathcal{F}_{\frac{i-1}{n}}\right)=0 .
$$

Next, let $N$ be any bounded martingale on $\left(\Omega, \mathcal{F},\left(\mathcal{F}_{t}\right)_{t \geq 0}, \mathbf{P}\right)$, which is orthogonal to $W$. For $j$ and $k$ fixed, we consider the martingale $M_{t}=\mathbf{E}\left(g\left(\beta_{i}^{n}\right)^{j k} \mid \mathcal{F}_{t}\right)$, for $t \geq \frac{i-1}{n}$. Since $W$ is an $\left(\mathcal{F}_{t}\right)$-Brownian motion, and since $\beta_{i}^{n}$ is a function of $\sigma_{(i-1) / n}$ and of $\Delta_{i}^{n} W$, we see that $\left(M_{t}\right)_{t \geq(i-1) / n}$ is also, conditionally on $\mathcal{F}_{(i-1) / n}$, a martingale w.r.t. the filtration which is generated by the process $W_{t}-W_{\frac{i-1}{n}}$. By the martingale representation theorem the process $M$ is thus of the form $M_{t}=M_{\frac{i-1}{n}}+\int_{\frac{i-1}{n}}^{t} \eta_{s} d W_{s}$ for an appropriate predictable process $\eta$. It follows that $M$ is orthogonal to the process $N_{t}^{\prime}=N_{t}-N_{\frac{i-1}{n}}$ (for $t \geq \frac{i-1}{n}$ ), or in other words the product $M N^{\prime}$ is an $\left(\mathcal{F}_{t}\right)_{t \geq \frac{i-1}{n}}$-martingale. Hence

$$
\begin{aligned}
\mathbf{E}\left(\Delta_{i}^{n} N g\left(\sqrt{n} \sigma_{\frac{i-1}{n}} \Delta_{i}^{n} W\right)^{j k} \mid \mathcal{F}_{\frac{i-1}{n}}\right) & =\mathbf{E}\left(\Delta_{i}^{n} N^{\prime} M_{i / n} \mid \mathcal{F}_{\frac{i-1}{n}}\right) \\
& =\mathbf{E}\left(\Delta_{i}^{n} N^{\prime} \Delta_{i}^{n} M \mid \mathcal{F}_{\frac{i-1}{n}}\right)=0,
\end{aligned}
$$

and thus

$$
\mathbf{E}\left(\zeta_{i}^{n} \Delta_{i}^{n} N \mid \mathcal{F}_{\frac{i-1}{n}}\right)=0 .
$$

If we put together (4.10), (4.11), (4.12) and (4.13), we deduce the result from Theorem IX.7.28 of [9].

Proof of Proposition 4.2. A simple computation shows that $U_{t}^{\prime n}=$ $\sum_{i=2}^{[n t]+1} \zeta_{i}^{n}+\gamma_{1}^{n}-\gamma_{[n t]+1}^{n}$, where

$$
\begin{gathered}
\zeta_{i}^{n}=\frac{1}{\sqrt{n}}\left(g\left(\beta_{i-1}^{n}\right)\left(h\left(\beta_{i-1}^{\prime n}\right)-\rho_{i-2}^{n}(h)\right)+\left(g\left(\beta_{i}^{n}\right)-\rho_{i-1}^{n}(g)\right) \rho_{i-1}^{n}(h)\right), \\
\gamma_{i}^{n}=\frac{1}{\sqrt{n}}\left(g\left(\beta_{i}^{n}\right)-\rho_{i-1}^{n}(g)\right) \rho_{i-1}^{n}(h) .
\end{gathered}
$$

We trivially have (4.10), while (4.12) and (4.13) (for any bounded martingale $N$ orthogonal to $W$ ) are proved exactly as in the previous proposition. We will write $\rho_{i-2, i-1}^{n}(g, h)=\int g\left(\sigma_{\frac{i-1}{n}} x\right) h\left(\sigma_{\frac{i-2}{n}} x\right) \rho(d x)$, where $\rho$ is the $\mathcal{N}\left(0, I_{d^{\prime}}\right)$ law. An easy computation shows that 


$$
\begin{aligned}
& \mathbf{E}\left(\zeta_{i}^{n, j k} \zeta_{i}^{n, j^{\prime} k^{\prime}} \mid \mathcal{F}_{\frac{i-1}{n}}\right) \\
& =\frac{1}{n} \sum_{l, l^{\prime}=1}^{d_{2}}\left[g\left(\beta_{i-1}^{n}\right)^{j l} g\left(\beta_{i-1}^{n}\right)^{j^{\prime} l^{\prime}}\left(\rho_{i-2}^{n}\left(h^{l k} h^{l^{\prime} k^{\prime}}\right)-\rho_{i-2}^{n}\left(h^{l k}\right) \rho_{i-2}^{n}\left(h^{l^{\prime} k^{\prime}}\right)\right)\right. \\
& \quad+g\left(\beta_{i-1}^{n}\right)^{j l} \rho_{i-1}^{n}\left(h^{l^{\prime} k^{\prime}}\right)\left(\rho_{i-2, i-1}^{n}\left(g^{j^{\prime} l^{\prime}}, h^{l k}\right)-\rho_{i-2}^{n}\left(h^{l k}\right) \rho_{i-1}^{n}\left(g^{j^{\prime} l^{\prime}}\right)\right) \\
& \quad+g\left(\beta_{i-1}^{n}\right)^{j^{\prime} l^{\prime}} \rho_{i-1}^{n}\left(h^{l k}\right)\left(\rho_{i-2, i-1}^{n}\left(g^{j l}, h^{l^{\prime} k^{\prime}}\right)-\rho_{i-2}^{n}\left(h^{l^{\prime} k^{\prime}}\right) \rho_{i-1}^{n}\left(g^{j l}\right)\right) \\
& \left.\quad+\rho_{i-1}^{n}\left(h^{l^{\prime} k^{\prime}}\right) \rho_{i-1}^{n}\left(h^{l k}\right)\left(\rho_{i-1}^{n}\left(g^{j l} g^{j^{\prime} l^{\prime}}\right)-\rho_{i-1}^{n}\left(g^{j l}\right) \rho_{i-1}^{n}\left(g^{j^{\prime} l^{\prime}}\right)\right)\right] .
\end{aligned}
$$

and thus by (4.8) and since the components of $g$ and $h$ satisfy (4.7) and are continuous and $\sigma$ is càdlàg (hence in particular $\rho_{i-2, i-1}^{n}(g, h)-\rho_{i-2}^{n}(g h)$ goes to 0 , uniformly in $i \leq[n t]+1)$, we get with the notation $(2.14)$ :

$$
\sum_{i=2}^{[n t]+1} \mathbf{E}\left(\zeta_{i}^{n, j k} \zeta_{i}^{n, j^{\prime} k^{\prime}} \mid \mathcal{F}_{\frac{i-1}{n}}\right) \rightarrow \int_{0}^{t} A\left(\sigma_{u}, g, h\right)^{j k, k^{\prime} j^{\prime}} d u
$$

Then exactly as in the previous proof we deduce that the processes $\sum_{i=1}^{[n t]} \zeta_{i}^{n}$ are $\mathrm{C}$-tight, and that they converge stably in law to the process $U(g, h)$ of (2.13) when further $g$ and $h$ are even.

On the other hand $\gamma_{i}^{n}$ is the transpose of the jump at time $i / n$ of the process $U^{n}$ of (4.2) when $\delta_{u}=\rho_{\sigma_{u}}\left(h^{*}\right)$ and $f=g^{*}$, so Proposition 4.1 yields $\sup _{i \leq[n t]}\left\|\gamma_{i}^{n}\right\| \stackrel{\mathbf{P}}{\longrightarrow} 0$ for any $t$ : hence the results.

\section{A second simplified problem}

So far $Y$ has played no role, but it will come in this section. Recalling (4.1), we set

$$
\xi_{i}^{n}=\sqrt{n} \Delta_{i}^{n} Y-\beta_{i}^{n}, \quad \xi_{i}^{\prime n}=\sqrt{n} \Delta_{i+1}^{n} Y-\beta_{i}^{\prime n} .
$$

Observe that

$$
\xi_{i}^{n}=\sqrt{n}\left(\int_{\frac{i-1}{n}}^{\frac{i}{n}} a_{u} d u+\int_{\frac{i-1}{n}}^{\frac{i}{n}}\left(\sigma_{u-}-\sigma_{\frac{i-1}{n}}\right) d W_{u}\right),
$$

and a similar equality for $\xi_{i}^{\prime n}$, with the integrals between $i / n$ and $(i+1) / n$. Then under (SH) we have for any $q \in[2, \infty)$, by Burkholder Inequality:

$$
\mathbf{E}\left(\left\|\sqrt{n} \Delta_{i}^{n} Y\right\|^{q}\right)+\mathbf{E}\left(\left\|\xi_{i}^{n}\right\|^{q}\right)+\mathbf{E}\left(\left\|\xi_{i}^{\prime n}\right\|^{q}\right) \leq C_{q} .
$$

We can now consider the processes $U^{n}(g, h)$ of (2.15): in view of (5.2), the conditional expectations in (2.15) are finite as soon as $g$ and $h$ have polynomial growth. 
Theorem 5.1. Under (SH) and if $g$ and $h$ are continuous with at most polynomial growth, the sequence of processes $U^{n}(g, h)$ of (2.15) is $C$-tight. If further $g$ and $h$ are even, it converges stably in law to the processes $U(g, h)$ of (2.13).

We first prove three lemmas. The first one is very simple:

Lemma 5.2. Let $\left(\zeta_{i}^{n}\right)$ be an array of random variables satisfying for all $t$ :

$$
\sum_{i=1}^{[n t]} \mathbf{E}\left(\left\|\zeta_{i}^{n}\right\|^{2} \mid \mathcal{F}_{\frac{i-1}{n}}\right) \stackrel{\mathbf{P}}{\longrightarrow} 0 .
$$

If further each $\zeta_{i}^{n}$ is $\mathcal{F}_{(i+1) / n}$-measurable, the array $\left(\zeta_{i}^{n}-\mathbf{E}\left(\zeta_{i}^{n} \mid \mathcal{F}_{(i-1) / n}\right)\right)$ is $A N$.

Proof. Of course the result is well known when $\zeta_{i}^{n}$ is $\mathcal{F}_{i / n}$-measurable. Otherwise, we set $\eta_{i}^{n}=\mathbf{E}\left(\zeta_{i}^{n} \mid \mathcal{F}_{i / n}\right)$. This new array satisfies also (5.3) and now $\eta_{i}^{n}$ is $\mathcal{F}_{i / n}-$ measurable: so the array $\left(\eta_{i}^{n}-\mathbf{E}\left(\eta_{i}^{n} \mid \mathcal{F}_{(i-1) / n}\right)\right)$ is AN.

Next, (5.3) and Lenglart's inequality (see e.g. I-3.30 in [9]) yield $\sum_{i=1}^{[n t]} \mathbf{E}\left(\left\|\zeta_{i}^{n}\right\|^{2} \mid \mathcal{F}_{i / n}\right) \stackrel{\mathbf{P}}{\longrightarrow}$ 0, so the afore mentionned well known result also yields that the array $\left(\zeta_{i}^{n}-\eta_{i}^{n}\right)$ is $\mathrm{AN}$, and the result follows.

Lemma 5.3. Under (SH) we have for all $t>0$ :

$$
\frac{1}{n} \sum_{i=1}^{[n t]} \mathbf{E}\left(\left\|\xi_{i}^{n}\right\|^{2}+\left\|\beta_{i+1}^{n}-\beta_{i}^{\prime n}\right\|^{2}\right) \rightarrow 0 .
$$

Proof. First, the boundedness of $a$ yields

$$
\mathbf{E}\left(\left\|\xi_{i}^{n}\right\|^{2}\right) \leq C\left(\frac{1}{n}+n \mathbf{E}\left(\int_{\frac{i-1}{n}}^{\frac{i}{n}}\left\|\sigma_{u-}-\sigma_{\frac{i-1}{n}}\right\|^{2} d u\right)\right) .
$$

We also trivially have

$$
\begin{aligned}
\mathbf{E}\left(\left\|\beta_{i+1}^{n}-\beta_{i}^{\prime n}\right\|^{2}\right) & \leq C \mathbf{E}\left(\left\|\sigma_{\frac{i}{n}}-\sigma_{\frac{i-1}{n}}\right\|^{2}\right) \\
& \leq C n \mathbf{E}\left(\int_{\frac{i-1}{n}}^{\frac{i}{n}}\left(\left\|\sigma_{u-}-\sigma_{\frac{i-1}{n}}\right\|^{2}+\left\|\sigma_{u-}-\sigma_{\frac{i}{n}}\right\|^{2}\right) d u\right) .
\end{aligned}
$$

Hence the left side of (5.4) is smaller than

$$
C\left(\frac{t}{n}+\int_{0}^{t} \mathbf{E}\left(\left\|\sigma_{u-}-\sigma_{[n u] / n}\right\|^{2}+\left\|\sigma_{u-}-\sigma_{([n u]+1) / n}\right\|^{2}\right) d u\right) .
$$

Since $\sigma$ is càdlàg, the expectation above goes to 0 for all $u$ except the fixed times of discontinuity of the process $\sigma$, that is for almost all $u$, and it stays 
bounded by a constant because of (SH): hence the result by Lebesgue's theorem.

For further reference, the third lemma is stated in a more general setting:

- $f$ and $k$ are functions on $\mathbf{R}^{d}$ satisfying (4.7);

- $\gamma_{i}^{n}, \gamma_{i}^{\prime n}, \gamma_{i}^{\prime \prime n}$ are $\mathbf{R}^{d_{-}}$-valued variables,

- $Z_{i}^{n}=1+\left\|\gamma_{i}^{n}\right\|+\left\|\gamma_{i}^{\prime n}\right\|+\left\|\gamma_{i}^{\prime \prime n}\right\|$ satisfies $\mathbf{E}\left(\left(Z_{i}^{n}\right)^{p}\right) \leq C_{p}$.

Lemma 5.4. Under (5.5) and if further $k$ is continuous and

$$
\frac{1}{n} \sum_{i=1}^{[n t]} \mathbf{E}\left(\left\|\gamma_{i}^{\prime n}-\gamma_{i}^{\prime \prime n}\right\|^{2}\right) \rightarrow 0
$$

then we have for all $t>0$ :

$$
\frac{1}{n} \sum_{i=1}^{[n t]} \mathbf{E}\left(f\left(\gamma_{i}^{n}\right)^{2}\left(k\left(\gamma_{i}^{\prime n}\right)-k\left(\gamma_{i}^{\prime \prime n}\right)\right)^{2}\right) \rightarrow 0 .
$$

Proof. Set $\theta_{i}^{n}=\left(f\left(\gamma_{i}^{n}\right)\left(k\left(\gamma_{i}^{\prime n}\right)-k\left(\gamma_{i}^{\prime \prime n}\right)\right)\right)^{2}$ and $m_{A}(\varepsilon)=\sup (|k(x)-k(y)|$ : $\|x-y\| \leq \varepsilon,\|x\| \leq A)$. For all $\varepsilon \in(0,1]$ and $A>1$ we have

$$
\begin{aligned}
\theta_{i}^{n} \leq & C\left(A^{2 p} m_{A}(\varepsilon)^{2}+A^{4 p} 1_{\left\{\left\|\gamma_{i}^{\prime n}-\gamma_{i}^{\prime \prime n}\right\|>\varepsilon\right\}}\right. \\
& \left.+\left(Z_{i}^{n}\right)^{4 p}\left(1_{\left\{\left\|\gamma_{i}^{n}\right\|>A\right\}}+1_{\left\{\left\|\gamma_{i}^{\prime n}\right\|>A\right\}}+1_{\left\{\left\|\gamma_{i}^{\prime \prime n}\right\|>A\right\}}\right)\right) \\
& \leq C\left(A^{2 p} m_{A}(\varepsilon)^{2}+\frac{A^{4 p}\left\|\gamma_{i}^{\prime n}-\gamma_{i}^{\prime \prime n}\right\|^{2}}{\varepsilon^{2}}+\frac{\left(Z_{i}^{n}\right)^{4 p+1}}{A}\right) .
\end{aligned}
$$

Then in view of (5.5) we get

$$
\frac{1}{n} \sum_{i=1}^{[n t]} \mathbf{E}\left(\theta_{i}^{n}\right) \leq C\left(A^{2 p} m_{A}(\varepsilon)^{2}+\frac{1}{A}+\frac{A^{4 p}}{n \varepsilon^{2}} \sum_{i=1}^{[n t]} \mathbf{E}\left(\left\|\gamma_{i}^{\prime n}-\gamma_{i}^{\prime \prime n}\right\|^{2}\right)\right)
$$

This holds for all $\varepsilon \in(0,1]$ and $A>1$. Since $m_{A}(\varepsilon) \rightarrow 0$ as $\varepsilon \rightarrow 0$, for every $A,(5.7)$ readily follows from $(5.6)$.

Proof of Theorem 5.1. In view of Proposition 4.2, it is clearly enough to prove that $U^{n}(g, h)-U^{\prime n} \stackrel{\mathbf{P}}{\longrightarrow} 0$. Set

$$
\zeta_{i}^{n}=\frac{1}{\sqrt{n}}\left(g\left(\sqrt{n} \Delta_{i}^{n} Y\right) h\left(\sqrt{n} \Delta_{i+1}^{n} Y\right)-g\left(\beta_{i}^{n}\right) h\left(\beta_{i}^{\prime n}\right)\right)
$$

and observe that $U^{n}(g, h)_{t}-U_{t}^{\prime n}=\sum_{i=1}^{[n t]}\left(\zeta_{i}^{n}-\mathbf{E}\left(\zeta_{i}^{n} \mid \mathcal{F}_{(i-1) / n}\right)\right)$ and that $\zeta_{i}^{n}$ is $\mathcal{F}_{(i+1) / n}$-measurable. Then by Lemma 5.2 it suffices to prove that 


$$
\sum_{i=1}^{[n t]} \mathbf{E}\left(\left\|\zeta_{i}^{n}\right\|^{2}\right) \rightarrow 0
$$

For proving (5.9) it is clearly enough to consider the case where both $g$ and $h$ are 1 -dimensional. Recalling $\sqrt{n} \Delta_{i}^{n} Y=\beta_{i}^{n}+\xi_{i}^{n}$, we then have

$$
\begin{aligned}
\left\|\zeta_{i}^{n}\right\|^{2} \leq & \frac{C}{n}\left(h\left(\sqrt{n} \Delta_{i+1}^{n} Y\right)^{2}\left(g\left(\beta_{i}^{n}+\xi_{i}^{n}\right)-g\left(\beta_{i}^{n}\right)\right)^{2}\right. \\
& \left.+g\left(\beta_{i}^{n}\right)^{2}\left(h\left(\beta_{i+1}^{n}+\xi_{i+1}^{n}\right)-h\left(\beta_{i+1}^{n}\right)\right)^{2}+g\left(\beta_{i}^{n}\right)^{2}\left(h\left(\beta_{i+1}^{n}\right)-h\left(\beta_{i}^{\prime n}\right)\right)^{2}\right) .
\end{aligned}
$$

Then (5.9) immediately follows from (4.6) and (5.2) and from Lemmas 5.3 and 5.4 .

\section{Proof of Theorem 2.1}

As stated in Section 2, we can and will assume (SH). We use the notation $\zeta_{i}^{n}$ of $(5.8)$, and set

$$
\eta_{i}^{n}=\mathbf{E}\left(g\left(\sqrt{n} \Delta_{i}^{n} Y\right) h\left(\sqrt{n} \Delta_{i+1}^{n} Y\right) \mid \mathcal{F}_{\frac{i-1}{n}}\right), \quad \eta_{i}^{\prime n}=\rho_{i-1}^{n}(g) \rho_{i-1}^{n}(h)
$$

and $V_{t}^{n}=\sum_{i=1}^{[n t]} \eta_{i}^{n}$ and $V_{t}^{\prime n}=\sum_{i=1}^{[n t]} \eta_{i}^{\prime n}$. Theorem 5.1 implies that $\frac{1}{n}\left(X^{n}(g, h)-V^{n}\right) \stackrel{\mathbf{P}}{\longrightarrow} 0$, and Riemann integrability yields $\frac{1}{n} V^{\prime n} \rightarrow X(g, h)$ pointwise in $\omega$ and locally uniformly in time. So we need to prove that $\frac{1}{n}\left(V^{n}-V^{\prime n}\right) \stackrel{\mathbf{P}}{\longrightarrow} 0$. Since $\eta_{i}^{n}-\eta_{i}^{\prime n}=\sqrt{n} \mathbf{E}\left(\zeta_{i}^{n} \mid \mathcal{F}_{(i-1) / n}\right)$, it clearly suffices to prove that

$$
\frac{1}{\sqrt{n}} \sum_{i=1}^{[n t]} \mathbf{E}\left(\left\|\zeta_{i}^{n}\right\|\right) \rightarrow 0
$$

By the Cauchy-Schwarz inequality, the left side of (6.1) is smaller than $\left(t \sum_{i=1}^{[n t]} \mathbf{E}\left(\left\|\zeta_{i}^{n}\right\|^{2}\right)\right)^{1 / 2}$ and thus (6.1) follows from (5.9).

\section{Technical preliminaries for Theorem 2.3}

As said before, for proving Theorem 2.3 we can and will assume (SH), and also (SH') when at least one of the components of $g$ or $h$ satisfies (K') instead of $(\mathrm{K})$. In fact, this theorem is deduced from Theorem 5.1, provided we can show that $\sqrt{n}\left(X^{n}(g, h)_{t}-U^{n}(g, h)_{t}\right)$ goes to 0 in probability, locally uniformly in $t$. This amounts to proving that the array

$$
\zeta_{i}^{n}=\frac{1}{\sqrt{n}} \mathbf{E}\left(g\left(\sqrt{n} \Delta_{i}^{n} Y\right) h\left(\sqrt{n} \Delta_{i+1}^{n} Y\right) \mid \mathcal{F}_{\frac{i-1}{n}}\right)-\sqrt{n} \int_{\frac{i-1}{n}}^{\frac{i}{n}} \rho_{\sigma_{u}}(g) \rho_{\sigma_{u}}(h) d u
$$


is AN. Obviously, we can work componentwise, and so we will assume w.l.o.g. that both $g$ and $h$ are 1 -dimensional (they still are functions on $\mathbf{R}^{d}$, though).

We have $\zeta_{i}^{n}=\zeta_{i}^{\prime n}+\zeta_{i}^{\prime \prime n}$, where

$$
\begin{aligned}
& \zeta_{i}^{\prime n}=\frac{1}{\sqrt{n}}\left(\mathbf{E}\left(g\left(\sqrt{n} \Delta_{i}^{n} Y\right) h\left(\sqrt{n} \Delta_{i+1}^{n} Y\right) \mid \mathcal{F}_{\frac{i-1}{n}}\right)\right. \\
&\left.-\mathbf{E}\left(g\left(\beta_{i}^{n}\right) \mid \mathcal{F}_{\frac{i-1}{n}}\right) \mathbf{E}\left(h\left(\beta_{i}^{\prime n}\right) \mid \mathcal{F}_{\frac{i-1}{n}}\right)\right), \\
& \zeta_{i}^{\prime \prime n}=\sqrt{n} \int_{\frac{i-1}{n}}^{\frac{i}{n}}\left(\rho_{\sigma_{u}}(g) \rho_{\sigma_{u}}(h)-\rho_{i-1}^{n}(g) \rho_{i-1}^{n}(h)\right) d u .
\end{aligned}
$$

So we are left to prove that both arrays $\left(\zeta_{i}^{\prime n}\right)$ and $\left(\zeta_{i}^{\prime \prime n}\right)$ are AN. For the second one this is relatively simple, but for the first one it is quite complicated, and we need to split the difference in (7.1) into a large number of terms, which are treated in different ways: this section is devoted to estimates for these various terms.

\subsection{Some notation}

First, we fix a sequence of numbers $\varepsilon_{n} \in(0,1]$ (which will be chosen later in such a way that $\left.\varepsilon_{n}^{2} n \geq 1\right)$, and we set $E_{n}=\left\{x \in E: \psi(x)>\varepsilon_{n}\right\}$. Then, recalling the product-matrix notation, under ( $\mathrm{SH} 1)$ we can introduce a (long) series of $\mathbf{R}^{d-\text { valued random variables: }}$

$$
\begin{aligned}
& \zeta(1)_{i}^{n}=\sqrt{n} \int_{\frac{i-1}{n}}^{\frac{i}{n}}\left(a_{u}-a_{\frac{i-1}{n}}\right) d u+\sqrt{n} \int_{\frac{i-1}{n}}^{\frac{i}{n}}\left(\int_{\frac{i-1}{n}}^{u} a_{s}^{\prime} d s\right. \\
& \left.+\int_{\frac{i-1}{n}}^{u}\left(\sigma_{s-}^{\prime}-\sigma_{\frac{i-1}{n}}^{\prime}\right) d W_{s}+\int_{\frac{i-1}{n}}^{u}\left(v_{s-}-v_{\frac{i-1}{n}}\right) d V_{s}\right) d W_{u}, \\
& \zeta(1)_{i}^{\prime n}=\sqrt{n}\left(\int_{\frac{i-1}{n}}^{\frac{i}{n}} a_{s}^{\prime} d s+\int_{\frac{i-1}{n}}^{\frac{i}{n}}\left(\sigma_{s-}^{\prime}-\sigma_{\frac{i-1}{n}}^{\prime}\right) d W_{s}\right. \\
& \left.+\int_{\frac{i-1}{n}}^{\frac{i}{n}}\left(v_{s-}-v_{\frac{i-1}{n}}\right) d V_{s}\right) \Delta_{i+1}^{n} W \\
& \zeta(2)_{i}^{n}=\sqrt{n}\left(\frac{1}{n} a_{\frac{i-1}{n}}+\sigma_{\frac{i-1}{n}}^{\prime} \int_{\frac{i-1}{n}}^{\frac{i}{n}}\left(W_{u}-W_{\frac{i-1}{n}}\right) d W_{u}\right. \\
& \left.+v_{\frac{i-1}{n}} \int_{\frac{i-1}{n}}^{\frac{i}{n}}\left(V_{u-}-V_{\frac{i-1}{n}}\right) d W_{u}\right), \\
& \zeta(2)_{i}^{\prime n}=\sqrt{n}\left(\sigma_{\frac{i-1}{n}}^{\prime} \Delta_{i}^{n} W+v_{\frac{i-1}{n}} \Delta_{i}^{n} V\right) \Delta_{i+1}^{n} W, \\
& \zeta(3)_{i}^{n}=\sqrt{n} \int_{\frac{i-1}{n}}^{\frac{i}{n}}\left(\int_{\frac{i-1}{n}}^{u} \int_{E_{n}^{c}} w(s-, x)(\mu-\nu)(d s, d x)\right) d W_{u}
\end{aligned}
$$




$$
\begin{aligned}
& \zeta(3)_{i}^{\prime n}=\sqrt{n}\left(\int_{\frac{i-1}{n}}^{\frac{i}{n}} \int_{E_{n}^{c}} w(s-, x)(\mu-\nu)(d s, d x)\right) \Delta_{i+1}^{n} W, \\
& \zeta(4)_{i}^{n}=-\sqrt{n} \int_{\frac{i-1}{n}}^{\frac{i}{n}}\left(\int_{\frac{i-1}{n}}^{u} \int_{E_{n}}\left(w(s-, x)-w\left(\frac{i-1}{n}, x\right)\right) \nu(d s, d x)\right) d W_{u}, \\
& \zeta(4)_{i}^{\prime n}=-\sqrt{n}\left(\int_{\frac{i-1}{n}}^{\frac{i}{n}} \int_{E_{n}}\left(w(s-, x)-w\left(\frac{i-1}{n}, x\right)\right) \nu(d s, d x)\right) \Delta_{i+1}^{n} W \\
& \zeta(5)_{i}^{n}=-\sqrt{n} \int_{\frac{i-1}{n}}^{\frac{i}{n}}\left(\int_{\frac{i-1}{n}}^{u} \int_{E_{n}} w\left(\frac{i-1}{n}, x\right) \nu(d s, d x)\right) d W_{u}, \\
& \zeta(5)_{i}^{\prime n}=-\sqrt{n}\left(\int_{\frac{i-1}{n}}^{\frac{i}{n}} \int_{E_{n}} w\left(\frac{i-1}{n}, x\right) \nu(d s, d x)\right) \Delta_{i+1}^{n} W, \\
& \zeta(6)_{i}^{n}=\sqrt{n} \int_{\frac{i-1}{n}}^{\frac{i}{n}}\left(\int_{\frac{i-1}{n}}^{u} \int_{E_{n}}\left(w(s-, x)-w\left(\frac{i-1}{n}, x\right)\right) \mu(d s, d x)\right) d W_{u}, \\
& \zeta(6)_{i}^{\prime n}=\sqrt{n}\left(\int_{\frac{i-1}{n}}^{\frac{i}{n}} \int_{E_{n}}\left(w(s-, x)-w\left(\frac{i-1}{n}, x\right)\right) \mu(d s, d x)\right) \Delta_{i+1}^{n} W, \\
& \zeta(7)_{i}^{n}=\sqrt{n} \int_{\frac{i-1}{n}}^{\frac{i}{n}}\left(\int_{\frac{i-1}{n}}^{u} \int_{E_{n}} w\left(\frac{i-1}{n}, x\right) \mu(d s, d x)\right) d W_{u}, \\
& \zeta(7)_{i}^{\prime n}=\sqrt{n}\left(\int_{\frac{i-1}{n}}^{\frac{i}{n}} \int_{E_{n}} w\left(\frac{i-1}{n},\right) \mu(d s, d x)\right) \Delta_{i+1}^{n} W .
\end{aligned}
$$

We also set

$$
\left.\begin{array}{ll}
\widehat{\xi}_{i}^{n}=\zeta(1)_{i}^{n}+\zeta(3)_{i}^{n}+\zeta(4)_{i}^{n}+\zeta(6)_{i}^{n}, & \widetilde{\xi}_{i}^{n}=\zeta(2)_{i}^{n}+\zeta(5)_{i}^{n}+\zeta(7)_{i}^{n} \\
\widehat{\xi}_{i}^{\prime \prime n}=\zeta(1)_{i}^{\prime n}+\zeta(3)_{i}^{\prime \prime}+\zeta(4)_{i}^{\prime n}+\zeta(6)_{i}^{\prime n}, & \\
\widetilde{\xi}_{i}^{\prime \prime n}=\zeta(2)_{i}^{\prime n}+\zeta(5)_{i}^{\prime \prime}+\zeta(7)_{i}^{\prime n} & \\
\widehat{\xi}_{i}^{\prime n}=\widehat{\xi}_{i+1}^{n}+\widehat{\xi}_{i}^{\prime \prime n}, & \widetilde{\xi}_{i}^{\prime n}=\widetilde{\xi}_{i+1}^{n}+\widetilde{\xi}_{i}^{\prime \prime n} .
\end{array}\right\}
$$

In view of (5.1), a tedious but simple computation shows that

$$
\sqrt{n} \Delta_{i}^{n} Y-\beta_{i}^{n}=\xi_{i}^{n}=\widehat{\xi}_{i}^{n}+\widetilde{\xi}_{i}^{n}, \quad \sqrt{n} \Delta_{i+1}^{n} Y-\beta_{i}^{\prime n}=\xi_{i}^{\prime n}=\widehat{\xi}_{i}^{n}+\widetilde{\xi}_{i}^{\prime n} .
$$

Next, we put $\varphi(\varepsilon)=\int_{\{\|\psi(x)\| \leq \varepsilon\}} \psi(x)^{2} F(d x)$, so that

$$
\begin{aligned}
& \varepsilon \downarrow 0 \Rightarrow \varphi(\varepsilon) \rightarrow 0 \\
& \theta \in[0,2] \quad \Rightarrow \quad \int_{\{\psi(x)>\varepsilon\}} \psi(x)^{\theta} F(d x) \leq \frac{C}{\varepsilon^{2-\theta}}, \\
& \left.\theta \geq 2 \Rightarrow \int_{\{\psi(x) \leq \varepsilon\}} \psi(x)^{\theta} F(d x) \leq \varphi(\varepsilon) \varepsilon^{\theta-2} .\right\}
\end{aligned}
$$


Finally, set

$$
\begin{aligned}
\alpha_{i}^{n, q}=\frac{1}{n^{q / 2}}+\mathbf{E} & \left(\left(n \int _ { \frac { i - 1 } { n } } ^ { \frac { i } { n } } \left(\left\|a_{u}-a_{\frac{i-1}{n}}\right\|^{2}+\left\|\sigma_{u-}^{\prime}-\sigma_{\frac{i-1}{n}}^{\prime}\right\|^{2}+\left\|v_{u-}-v_{\frac{i-1}{n}}\right\|^{2}\right.\right.\right. \\
& \left.\left.\left.+\int_{E_{n}}\left\|w(u-, x)-w\left(\frac{i-1}{n}, x\right)\right\|^{2} F(d x)\right) d u\right)^{q / 2}\right), \quad(7.6
\end{aligned}
$$

\subsection{Estimates for $\zeta(k)_{j}^{n}$ and $\zeta(k)_{j}^{\prime n}$}

Here we estimate moments of the variables $\zeta(k)_{i}^{n}$ and $\zeta(k)_{i}^{\prime n}$. A repeated use of the Hölder and Burkholder inequalities gives us for $q \geq 2$, and under (SH1):

$$
\left.\begin{array}{l}
\mathbf{E}\left(\left\|\zeta(1)_{i}^{n}\right\|^{q}\right)+\mathbf{E}\left(\left\|\zeta(1)_{i}^{\prime n}\right\|^{q}\right) \leq C_{q} \alpha_{i}^{n, q} / n^{q / 2}, \\
\mathbf{E}\left(\left\|\zeta(2)_{i}^{n}\right\|^{q}\right)+\mathbf{E}\left(\left\|\zeta(2)_{i}^{\prime n}\right\|^{q}\right) \leq C_{q} / n^{q / 2} .
\end{array}\right\}
$$

Lemma 7.1. Under (SH1), and for any even integer $q \geq 2$, we have

$$
\mathbf{E}\left(\left\|\zeta(3)_{i}^{n}\right\|^{q}\right)+\mathbf{E}\left(\left\|\zeta(3)_{i}^{\prime n}\right\|^{q}\right) \leq C_{q} \varphi\left(\varepsilon_{n}\right) \frac{\varepsilon_{n}^{q-2}}{n} .
$$

Proof. Apply the Hölder and Burkholder inequalities repeatedly to get

$$
\begin{aligned}
\mathbf{E}\left(\left\|\zeta(3)_{i}^{n}\right\|^{q}\right) & \leq C_{q} \mathbf{E}\left(\left(n \int_{\frac{i-1}{n}}^{\frac{i}{n}}\left\|\int_{\frac{i-1}{n}}^{u} \int_{E_{n}^{c}} w(s, x)(\mu-\nu)(d s, d x)\right\|^{2} d u\right)^{q / 2}\right) \\
& \leq C_{q} n \int_{\frac{i-1}{n}}^{\frac{i}{n}} \mathbf{E}\left(\left\|\int_{\frac{i-1}{n}}^{u} \int_{E_{n}^{c}} w(s, x)(\mu-\nu)(d s, d x)\right\|^{q} d u\right) \\
& \leq C_{q} n \int_{\frac{i-1}{n}}^{\frac{i}{n}} \mathbf{E}\left(\left(\int_{\frac{i-1}{n}}^{u} \int_{E_{n}^{c}}\|w(s, x)\|^{2} \mu(d s, d x)\right)^{q / 2}\right) d u \\
& \leq C_{q} \mathbf{E}\left(\left(\int_{\frac{i-1}{n}}^{\frac{i}{n}} \int_{E_{n}^{c}} \psi(x)^{2} \mu(d s, d x)\right)^{q / 2}\right): \\
& =\mathbf{E}\left(\left(Z_{\frac{i}{n}}^{n}-Z_{\frac{i-1}{n}}^{n}\right)^{q / 2}\right)
\end{aligned}
$$

where $Z_{t}^{n}=\int_{0}^{t} \int_{E_{n}^{c}} \psi(x)^{2} \mu(d s, d x)$ is an increasing pure jump Lévy process, whose Laplace transform is

$$
\lambda \mapsto \mathbf{E}\left(e^{-\lambda\left(Z_{s+t}^{n}-Z_{s}^{n}\right)}\right)=\exp t \int_{E_{n}^{c}}\left(e^{-\lambda \psi(x)^{2}}-1\right) F(d x) .
$$

We compute the $q / 2-$ moment of $Z_{s+t}^{n}-Z_{s}^{n}$ by differentiating $q / 2$ times the Laplace transform at 0 : this is the sum, over all choices $u_{1}, \ldots, u_{k}$ of positive 
integers with $\sum_{i=1}^{k} u_{i}=q / 2$, of suitable constants times the product for all $i=1, \ldots, k$ of the terms $t \int_{E_{n}^{c}} \psi(x)^{2 u_{i}} F(d x)$; moreover this term is smaller than $t \varepsilon_{n}^{2 u_{i}-2} \varphi\left(\varepsilon_{n}\right)$. Since further $\varepsilon_{n} \leq 1$ and $\varphi(1)<\infty$, we deduce that

$$
\mathbf{E}\left(\left(Z_{s+t}^{n}-Z_{s}^{n}\right)^{q / 2}\right) \leq C_{q} \varphi\left(\varepsilon_{n}\right) \sum_{k=1}^{q / 2} t^{k} \varepsilon_{n}^{q-2 k} \leq C_{q} \varphi\left(\varepsilon_{n}\right)\left(t \varepsilon_{n}^{q-2}+t^{q / 2}\right) .
$$

We deduce (7.8) for $\zeta(3)_{i}^{n}$ (recall $n \varepsilon_{n}^{2} \geq 1$ ), and the same holds for $\zeta(3)_{i}^{\prime n}$.

Lemma 7.2. Under (SH1), for any $q>2$ we have

$$
\mathbf{E}\left(\left\|\zeta(4)_{i}^{n}\right\|^{q}\right)+\mathbf{E}\left(\left\|\zeta(4)_{i}^{\prime n}\right\|^{q}\right)+\mathbf{E}\left(\left\|\zeta(5)_{i}^{n}\right\|^{q}\right)+\mathbf{E}\left(\left\|\zeta(5)_{i}^{\prime n}\right\|^{q}\right) \leq \frac{C_{q}}{\varepsilon_{n}^{q} n^{q}} .
$$

Proof. Applying the Hölder and Burkholder inequalities and $\|w(s, x)\| \leq$ $\psi(x)$ yields for $j=4,5$ :

$$
\begin{aligned}
& \mathbf{E}\left(\left\|\zeta(j)_{i}^{n}\right\|^{q}+\left\|\zeta(j)_{i}^{\prime n}\right\|^{q}\right) \leq \\
& \quad \leq C_{q} \mathbf{E}\left(\left(n \int_{\frac{i-1}{n}}^{\frac{i}{n}}\left(\int_{\frac{i-1}{n}}^{u} \int_{E_{n}} \psi(x) \nu(d s, d x)\right)^{2} d u\right)^{q / 2}\right) \\
& \quad \leq C_{q}\left(\int_{\frac{i-1}{n}}^{\frac{i}{n}} d s \int_{E_{n}} \psi(x) F(d x)\right)^{q} \leq \frac{C_{q}}{n^{q}}\left(\int_{E_{n}} \psi(x) F(d x)\right)^{q} .
\end{aligned}
$$

The result readily follows from (7.5).

For $\zeta(j)_{i}^{n}$ and $\zeta(j)_{i}^{\prime n}$ with $j=6,7$ the analogous estimates are not quite enough for our purposes, and we need a bit more. Below, we consider a pair $(r, B)$, where $r \in(0,1]$ and $B$ is a closed subset of $\mathbf{R}^{d}$, with Lebesgue measure 0 , and such that (2.11) holds when $r<1$ and that $r=1$ if $B=\emptyset$. Let also

$$
\left.\begin{array}{l}
r=1 \quad \Rightarrow \quad \widehat{\gamma}_{i}^{n}=1 \\
r<1 \quad \Rightarrow \quad \widehat{\gamma}_{i}^{n}=1+\frac{1}{d\left(\gamma_{i}^{n}, B\right)}, \quad \text { with either } \gamma_{i}^{n}=\beta_{i}^{n} \text { or } \gamma_{i}^{n}=\beta_{i}^{\prime n}
\end{array}\right\}
$$

Lemma 7.3. Under (SH1) and the previous assumptions, and if further (SH') holds whenever $r<1$, for any $q \in(1,2)$ and $l \in[0,1)$ we can find $u>1$ (depending on $q$ and $l$ ) such that

$$
\left.\begin{array}{l}
\mathbf{E}\left(\left\|\zeta(6)_{i}^{n}\right\|^{q}\left(\widehat{\gamma}_{i}^{n}\right)^{l}\right)+\mathbf{E}\left(\left\|\zeta(6)_{i}^{\prime n}\right\|^{q}\left(\widehat{\gamma}_{i}^{n}\right)^{l}\right) \leq \frac{C_{l, q}\left(\alpha_{i}^{n, 2}\right)^{1 / u}}{n^{q / 2}}, \\
\mathbf{E}\left(\left\|\zeta(7)_{i}^{n}\right\|^{q}\left(\widehat{\gamma}_{i}^{n}\right)^{l}\right)+\mathbf{E}\left(\left\|\zeta(7)_{i}^{\prime n}\right\|^{q}\left(\widehat{\gamma}_{i}^{n}\right)^{l}\right) \leq \frac{C_{l, q}}{n^{q / 2}} .
\end{array}\right\}
$$


Proof. We set $M_{i}^{n}=\sup _{s \in[(i-1) / n, i / n]}\left\|W_{s}-W_{(i-1) / n}\right\|$ and $w_{n}(s, x)=$ $w(s-, x)-w\left(\frac{i-1}{n}, x\right)$ for $\frac{i-1}{n}<s \leq \frac{i}{n}$, and

$$
Z_{t}^{n}=\int_{0}^{t} \int_{E_{n}} \psi(x) \mu(d s, d x), \quad Z_{t}^{\prime n}=\int_{0}^{t} \int_{E_{n}}\left\|w_{n}(s, x)\right\| \mu(d s, d x) .
$$

Observe that $Z^{n}$ and $Z^{\prime n}$ are nondecreasing, piecewise constant, and $Z_{t}^{\prime n}-$ $Z_{s}^{\prime n} \leq 2\left(Z_{t}^{n}-Z_{s}^{n}\right)$ whenever $s<t$. Then

$$
\left\|\zeta(6)_{i}^{n}\right\| \leq C \sqrt{n} M_{i}^{n}\left(Z_{\frac{i}{n}}^{\prime n}-Z_{\frac{i-1}{n}}^{\prime n}\right) .
$$

Set $u^{\prime}=\frac{1}{2}\left(1+\frac{1}{l} \bigwedge \frac{1}{q-1}\right)$, which satisfies $u^{\prime}>1$ because $l<1$ and $q \in(1,2)$. With $\delta_{i}^{n}=\left(\sqrt{n} M_{i}^{n}\right)^{u^{\prime} q}\left(\widehat{\gamma}_{i}^{n}\right)^{u^{\prime} l}$ we then have (since $u^{\prime}>1$ and $\left.u^{\prime} q-u^{\prime}+1>0\right)$ :

$$
\left\|\zeta(6)_{i}^{n}\right\|^{q}\left(\widehat{\gamma}_{i}^{n}\right)^{l} \leq C_{q}\left(\delta_{i}^{n}\left(Z_{\frac{i}{n}}^{n}-Z_{\frac{i-1}{n}}^{n}\right)^{u^{\prime} q-u^{\prime}+1}\right)^{\frac{1}{u^{\prime}}}\left(Z_{\frac{i}{n}}^{\prime n}-Z_{\frac{i-1}{n}}^{\prime n}\right)^{\frac{u^{\prime}-1}{u^{\prime}}},
$$

and Hölder's inequality yields

$$
\begin{aligned}
& \mathbf{E}\left(\left\|\zeta(6)_{i}^{n}\right\|^{q}\left(\widehat{\gamma}_{i}^{n}\right)^{l}\right) \\
& \quad \leq C_{q}\left(\mathbf{E}\left(\delta_{i}^{n}\left(Z_{\frac{i}{n}}^{n}-Z_{\frac{i-1}{n}}^{n}\right)^{u^{\prime} q-u^{\prime}+1}\right)\right)^{\frac{1}{u^{\prime}}}\left(\mathbf{E}\left(Z_{\frac{i}{n}}^{\prime n}-Z_{\frac{i-1}{n}}^{\prime n}\right)\right)^{\frac{u^{\prime}-1}{u^{\prime}}} .
\end{aligned}
$$

Now, if we combine (2.11) and (3.6), we see that when $r<1$ (so (SH') holds) the variable $d\left(\gamma_{i}^{n}, B\right)$ has a conditional law knowing $\mathcal{F}_{(i-1) / n}$ which has a density which is bounded uniformly in $n, i$ and $\omega$, so $\mathbf{E}\left(\left(\widehat{\gamma}_{i}^{n}\right)^{s} \mid \mathcal{F}_{(i-1) / n}\right)$ is bounded by a constant $C_{s}$ for all $s \in[0,1)$, whether $r=1$ or $r<1$. Also, $\mathbf{E}\left(\left(\sqrt{n} M_{i}^{n}\right)^{p} \mid \mathcal{F}_{(i-1) / n}\right) \leq C_{q}$ for all $p>0$. Then by Hölder's inequality we get $\mathbf{E}\left(\delta_{i}^{n} \mid \mathcal{F}_{(i-1) / n}\right) \leq C_{q, l}$. Since further the variable $Z_{i / n}^{n}-Z_{(i-1) / n}^{n}$ is independent of $\delta_{i}^{n}$, conditionally on $\mathcal{F}_{\frac{i-1}{n}}$, we deduce

$$
\mathbf{E}\left(\delta_{i}^{n}\left(Z_{\frac{i}{n}}^{n}-Z_{\frac{i-1}{n}}^{n}\right)^{u^{\prime} q-u^{\prime}+1}\right) \leq C_{q, l} \mathbf{E}\left(\left(Z_{\frac{i}{n}}^{n}-Z_{\frac{i-1}{n}}^{n}\right)^{u^{\prime} q-u^{\prime}+1}\right) .
$$

Next, we estimate the moments of $Z^{n}$ and $Z^{\prime n}$. Observe that $Z^{\prime n}=A^{\prime n}+$ $N^{\prime n}$, where

$$
A_{t}^{\prime n}=\int_{0}^{t} \int_{E_{n}}\left\|w_{n}(s, x)\right\| \nu(d s, d x), \quad N^{\prime n}=\int_{0}^{t} \int_{E_{n}}\left\|w_{n}(s, x)\right\|(\mu-\nu)(d s, d x) .
$$

On the one hand, since $F\left(E_{n}\right) \leq C / \varepsilon_{n}^{2}$ by $(7.5)$ and $n \varepsilon_{n}^{2} \geq 1$,

$$
\begin{aligned}
\left(A_{\frac{i}{n}}^{\prime n}-A_{\frac{i-1}{n}}^{\prime n}\right)^{2} & \leq \frac{1}{n} \int_{\frac{i-1}{n}}^{\frac{i}{n}} d s\left(\int_{E_{n}}\left\|w_{n}(s, x)\right\| F(d x)\right)^{2} \\
& \leq \frac{1}{n} \int_{\frac{i-1}{n}}^{\frac{i}{n}} d s F\left(E_{n}\right) \int_{E_{n}}\left\|w_{n}(s, x)\right\|^{2} F(d x) \\
& \leq \int_{\frac{i-1}{n}}^{\frac{i}{n}} d s \int_{E_{n}}\left\|w_{n}(s, x)\right\|^{2} F(d x) .
\end{aligned}
$$


On the other hand $N^{\prime n}$ is a square-integrable martingale, and thus

$$
\mathbf{E}\left(\left(N_{\frac{i}{n}}^{\prime n}-N_{\frac{i-1}{n}}^{\prime n}\right)^{2}\right) \leq \mathbf{E}\left(\int_{\frac{i-1}{n}}^{\frac{i}{n}} d s \int_{E_{n}}\left\|w_{n}(s, x)\right\|^{2} F(d x)\right),
$$

and thus

$$
\mathbf{E}\left(\left(Z_{\frac{i}{n}}^{\prime n}-Z_{\frac{i-1}{n}}^{\prime n}\right)^{2}\right) \leq \frac{C \alpha_{i}^{n, 2}}{n} .
$$

If we replace $\left\|w_{n}(s, x)\right\|$ by $\psi(x)$, we obtain in a similar fashion

$$
\mathbf{E}\left(\left(Z_{\frac{i}{n}}^{n}-Z_{\frac{i-1}{n}}^{n}\right)^{2}\right) \leq \frac{C}{n} .
$$

Then if we combine (7.13), (7.14), (7.15) and (7.16), and since $u^{\prime} q-u^{\prime}+1 \leq 2$, we obtain the result for $\zeta(6)_{i}^{n}$, with $u=\frac{2 u^{\prime}}{u^{\prime}-1}>1$, and the proof for $\zeta(6)_{i}^{\prime n}$ is similar. Finally if we replace $w_{n}$ by $w$ (then $\alpha_{i}^{n, 2}$ is replaced by a constant), we get the result for $\zeta(7)_{i}^{n}$ and $\zeta(7)_{i}^{\prime n}$.

\subsection{Estimates for the variables of (7.3)}

Here we derive estimates on the variables defined in (7.3). Below, the pair $(B, r)$ and the variable $\widehat{\gamma}_{i}^{n}$ are like in Lemma 7.3. We also consider positive random variables $Z_{i}^{n}$ which satisfy

$$
\mathbf{E}\left(\left(Z_{i}^{n}\right)^{q}\right) \leq C_{q} \quad \forall q \geq 2 .
$$

Observe that $\xi_{i}^{n}$ and $\xi_{i}^{\prime n}$ do not depend on the sequence $\varepsilon_{n}$, but $\widehat{\xi}_{i}^{n}$ and $\widehat{\xi}_{i}^{\prime n}$ do. Remember also the variables $\alpha_{i}^{n, q}$ defined ibn (7.6).

Lemma 7.4. Assume (SH1) and (SH') and (7.11) and (7.17). Let $p \geq 2$ and $l \in(0,1)$. Then if $\theta \in(1,2)$ we have

$$
\mathbf{E}\left(\left(Z_{i}^{n}\right)^{p}\left\|\widetilde{\xi}_{i}^{n}\right\|^{\theta}\left(\widehat{\gamma}_{i}^{n}\right)^{l}\right)+\mathbf{E}\left(\left(Z_{i}^{n}\right)^{p}\left\|\widetilde{\xi}_{i}^{\prime n}\right\|^{\theta}\left(\widehat{\gamma}_{i}^{n}\right)^{l}\right) \leq \frac{C_{p, \theta, l}}{n^{\theta / 2}},
$$

Moreover one can find a sequence $\varepsilon_{n}>0$ with $n \varepsilon_{n}^{2} \geq 1$ and a sequence $z_{n}>0$ with $z_{n} \rightarrow 0$, both sequences depending on l only, and also two numbers $q, q^{\prime} \geq$ 1 depending on $l$ only, such that

$$
\left.\begin{array}{r}
\mathbf{E}\left(\left(Z_{i}^{n}\right)^{p}\left\|\widehat{\xi}_{i}^{n}\right\|\left(\widehat{\gamma}_{i}^{n}\right)^{l}\right) \leq \frac{C_{p, l}}{\sqrt{n}}\left(z_{n}+\left(\alpha_{i}^{n, q}\right)^{1 / q}+\left(\alpha_{i}^{n, 2}\right)^{1 / q^{\prime}}\right), \\
\left.\mathbf{E}\left(\left(Z_{i}^{n}\right)^{p}\left\|\widehat{\xi}_{i}^{\prime n}\right\|\left(\widehat{\gamma}_{i}^{n}\right)^{l}\right)\right) \leq \frac{C_{p, l}}{\sqrt{n}}\left(z_{n}+\left(\alpha_{i}^{n, q}\right)^{1 / q}+\left(\alpha_{i+1}^{n, q}\right)^{1 / q}\right. \\
\left.+\left(\alpha_{i}^{n, 2}\right)^{1 / q^{\prime}}+\left(\alpha_{i+1}^{n, 2}\right)^{1 / q^{\prime}}\right) .
\end{array}\right\}
$$


Proof. We prove (7.18) and (7.19) for $\xi_{i}^{n}$ and $\widehat{\xi}_{i}^{n}$ only, the proofs for $\xi_{i}^{\prime n}$ and $\widehat{\xi}_{i}^{\prime n}$ being similar. We have seen in the proof of Lemma 7.3 that, by (7.11),

$$
s \in[0,1) \quad \Rightarrow \quad \mathbf{E}\left(\left(\widehat{\gamma}_{i}^{n}\right)^{s}\right) \leq C_{s} .
$$

Although $\xi_{i}^{n}$ does not depend on the sequence $\varepsilon_{n}$, we need to introduce a suitable sequence $\varepsilon_{n}$ to prove (7.18): so we prove (7.18) and (7.19) simultaneously, with some fixed $\theta \in[1,2)$ for the first result, and with $\theta=1$ for the second one. If $t=\frac{1}{2}\left(1+\frac{1}{l} \wedge \frac{2}{\theta}\right)$, by (7.17) and Hólder's inequality we get

$$
\left.\begin{array}{l}
\mathbf{E}\left(\left(Z_{i}^{n}\right)^{p}\left\|\xi_{i}^{n}\right\|^{\theta}\left(\widehat{\gamma}_{i}^{n}\right)^{l}\right) \leq C_{p, \theta, l}\left(\mathbf{E}\left(\left\|\xi_{i}^{n}\right\|^{t \theta}\left(\widehat{\gamma}_{i}^{n}\right)^{t l}\right)\right)^{1 / t}, \\
\mathbf{E}\left(\left(Z_{i}^{n}\right)^{p}\left\|\widehat{\xi}_{i}^{n}\right\|\left(\widehat{\gamma}_{i}^{n}\right)^{l}\right) \leq C_{p, l}\left(\mathbf{E}\left(\left\|\widehat{\xi}_{i}^{n}\right\|^{t}\left(\widehat{\gamma}_{i}^{n}\right)^{t l}\right)\right)^{1 / t} .
\end{array}\right\}
$$

Next, let $s$ be the biggest number in $(1,1 / t l)$ such that its conjugate exponent $s^{\prime}$ is of the form $s^{\prime}=2 m / t \theta$ for some $m \in \mathbf{N}$ with $m \geq 2$, and put $q=s^{\prime} t \theta$. Note that $s^{\prime}$ and $q$ depend on $\theta$ and $l$ only. The set $\left\{y>0: y^{q} \varphi(y / \sqrt{n}) \leq 1\right\}$ is an open or semi-open interval whose left end point is 0 , and whose right end point is denoted by $a_{n}^{\prime}$, and since $\varphi(y) \rightarrow 0$ as $y \rightarrow 0$ it is clear that $a_{n}^{\prime} \rightarrow \infty$. At this point, we set $a_{n}=1 \bigvee\left(a_{n}^{\prime}-1 / n\right)$ : then $a_{n} \rightarrow \infty$, and for all $n$ big enough $a_{n}<a_{n}^{\prime}$ and thus $a_{n}^{q} \varphi\left(a_{n} / \sqrt{n}\right) \leq 1$. Then we choose the sequence $\varepsilon_{n}$ as $\varepsilon_{n}=a_{n} / \sqrt{n}$, thus $n \varepsilon_{n}^{2} \geq 1$. Observe that both sequences $\varepsilon_{n}$ and $a_{n}$ only depend on $\theta$ and $l$.

Now we apply (7.8) and (7.9) with $q$ and $\varepsilon_{n}$ as above, plus (7.20) and Hölder's inequality, to get

$$
\left.\begin{array}{l}
\left(\mathbf{E}\left(\left\|\zeta(3)_{i}^{n}\right\|^{t \theta}\left(\widehat{\gamma}_{i}^{n}\right)^{t l}\right)^{1 / t} \leq \frac{C_{\theta, l} \varphi\left(\varepsilon_{n}\right)^{1 / s^{\prime} t} a_{n}^{\theta-2 / s^{\prime} t}}{n^{\theta / 2}} \leq \frac{C_{\theta, l}}{n^{\theta / 2} a_{n}^{2 / s^{\prime} t}} \leq \frac{C_{\theta, l}}{n^{\theta / 2}},\right. \\
\left(\mathbf{E}\left(\left\|\zeta(4)_{i}^{n}\right\|^{t \theta}\left(\widehat{\gamma}_{i}^{n}\right)^{t l}\right)^{1 / t}+\left(\mathbf{E}\left(\left\|\zeta(5)_{i}^{n}\right\|^{t \theta}\left(\widehat{\gamma}_{i}^{n}\right)^{t l}\right)^{1 / t} \leq \frac{C_{\theta, l}}{n^{\theta / 2} a_{n}^{\theta}} \leq \frac{C_{\theta, l}}{n^{\theta / 2}} .\right.\right.
\end{array}\right\}
$$

In a similar way, (7.20) and (7.7) and Hólder's inequality give (with the same $q$ as above):

$$
\left.\begin{array}{l}
\left(\mathbf{E}\left(\left\|\zeta(1)_{i}^{n}\right\|^{t \theta}\left(\widehat{\gamma}_{i}^{n}\right)^{t l}\right)^{1 / t} \leq \frac{C_{\theta, l}\left(\alpha_{i}^{n, q}\right)^{\theta / q}}{n^{\theta / 2}},\right. \\
\left(\mathbf{E}\left(\left\|\zeta(2)_{i}^{n}\right\|^{t \theta}\left(\widehat{\gamma}_{i}^{n}\right)^{t l}\right)^{1 / t} \leq \frac{C_{\theta, l} .}{n^{\theta / 2}} .\right.
\end{array}\right\}
$$

Finally applying (7.12) and $t \theta<2$ yields

$$
\left.\begin{array}{l}
\left(\mathbf{E}\left(\left\|\zeta(6)_{i}^{n}\right\|^{t \theta}\left(\widehat{\gamma}_{i}^{n}\right)^{t l}\right)^{1 / t} \leq \frac{C_{\theta, l}\left(\alpha_{i}^{n, 2}\right)^{1 / q^{\prime}}}{n^{\theta / 2}},\right. \\
\left(\mathbf{E}\left(\left\|\zeta(7)_{i}^{n}\right\|^{t \theta}\left(\widehat{\gamma}_{i}^{n}\right)^{t l}\right)^{1 / t} \leq \frac{C_{\theta, l}}{n^{\theta / 2}}\right.
\end{array}\right\}
$$

for some $q^{\prime}>1$ depending on $t \theta$ and $t l$, hence on $\theta$ and $l$ only.

Then if we put together (7.21), (7.22), (7.23) and (7.24), and in view of (7.3) and (7.4), we readily get (7.18), and also (7.19) with $z_{n}=a_{n}^{-2 / s^{\prime} t}+a_{n}^{-1}$ (note that for $(7.19)$ we take $\theta=1)$. 


\subsection{Final estimates}

The previous subsection gave us estimates on the variables of (7.3), which in view of (7.4) are the building blocks for obtaining the difference occuring in (7.1). Now we procees to give estimates for this difference itself. We start with a lemma about the variables of (7.6).

Lemma 7.5. Under (SH1) we have for all $q \geq 2$ and $q^{\prime} \geq 1$ and $t>0$ :

$$
\alpha_{i}^{n, q} \leq C_{q}, \quad \frac{1}{n} \sum_{i=1}^{[n t]}\left(\alpha_{i}^{n, q}\right)^{1 / q^{\prime}} \rightarrow 0 .
$$

Proof. We can of course forget about the term $1 / n^{q / 2}$ in (7.6), whereas the first part of (7.25) is obvious. For the second part we set

$$
\begin{array}{r}
\gamma_{n}(u)=\left\|a_{u}-a_{[n u] / n}\right\|^{2}+\left\|\sigma_{u-}^{\prime}-\sigma_{[n u] / n}^{\prime}\right\|^{2}+\left\|v_{u-}-v_{[n u] / n}\right\|^{2} \\
+\int_{E}\left\|w(u-, x)-w\left(\frac{i-1}{n}, x\right)\right\|^{2} F(d x) .
\end{array}
$$

Then the Hölder inequality yields

$$
\begin{aligned}
\frac{1}{n} \sum_{i=1}^{[n t]}\left(\alpha_{i}^{n, q}\right)^{1 / q^{\prime}} & \leq \frac{[n t]}{n}\left(\frac{1}{[n t]} \sum_{i=1}^{[n t]} \mathbf{E}\left(\left(n \int_{\frac{i-1}{n}}^{\frac{i}{n}} \gamma_{n}(u) d u\right)^{q / 2}\right)\right)^{1 / q^{\prime}} \\
& \leq \frac{[n t]}{n}\left(\frac{1}{[n t]} \sum_{i=1}^{[n t]} \mathbf{E}\left(n \int_{\frac{i-1}{n}}^{\frac{i}{n}} \gamma_{n}(u)^{q / 2} d u\right)\right)^{1 / q^{\prime}} \\
& \leq t^{\frac{q^{\prime}-1}{q^{\prime}}}\left(\mathbf{E}\left(\int_{0}^{t} \gamma_{n}(u)^{q / 2} d u\right)\right)^{1 / q^{\prime}}
\end{aligned}
$$

Since $\gamma_{n}$ is uniformly bounded and converges pointwise to 0 , we get the result.

Let us now introduce a list of growth or smoothness assumptions on a real-valued function $f$ on $\mathbf{R}^{d}$, with complement (4.7). Below, $C>0$ and $p \geq 2$ are suitable constants, and the pair $(B, r)$ is given, with the properties stated before (7.11). We list some conditions, for which we assume that $f$ is differentiable on the complement $B^{c}$. Below, each $\Psi_{A, \varepsilon}$ is an increasing continuous function on $\mathbf{R}_{+}$with $\Psi_{A, \varepsilon}(0)=0$.

$$
\begin{gathered}
x \in B^{c} \quad \Rightarrow \quad|\nabla f(x)| \leq C\left(1+\|x\|^{p}\right)\left(1+\frac{1}{d(x, B)^{1-r}}\right), \\
x, y \in \mathbf{R}^{d} \quad \Rightarrow \quad|f(x+y)-f(x)| \leq C\left(1+\|x\|^{p}+\|y\|^{p}\right)\|y\|^{r},
\end{gathered}
$$




$$
\begin{aligned}
\|x\| \leq A,\|y\| & \leq \varepsilon^{\prime}<\varepsilon<d(x, B) \Rightarrow\|\nabla f(x+y)-\nabla f(x)\| \leq \Psi_{A, \varepsilon}\left(\varepsilon^{\prime}\right) \\
0<\|y\| & \leq \frac{d(x, B)}{2} \\
& \Longrightarrow\|\nabla f(x+y)-\nabla f(x)\| \leq C\left(1+\|x\|^{p}+\|y\|^{p}\right) \frac{\|y\|}{d(x, B)^{2-r}} .
\end{aligned}
$$

The connections with our assumptions $(\mathrm{K})$ and $\left(\mathrm{K}^{\prime}\right)$ are as follows (with $B$ and $r$ identical in (K') and above, or $B=\emptyset$ and $r=1$ in the case of $(\mathrm{K}))$ :

$$
(\mathrm{K}) \text {, or (K') with } r=1 \Rightarrow(4.7),(7.26),(7.27) \text { and (7.28), }
$$

$$
\text { (K') with } r<1 \Rightarrow(4.7),(7.26),(7.27) \text { and (7.29) }
$$

Next, we consider the setting of (5.5), with $k$ is differentiable on $B^{c}$. We let $\gamma_{i}^{\prime \prime n}$ be either $\beta_{i}^{n}$ or $\beta_{i}^{\prime n}$, and we introduce the following subsets of $\Omega$ :

$$
A_{i}^{n}=\left\{\left\|\gamma_{i}^{\prime n}-\gamma_{i}^{\prime \prime n}\right\|>d\left(\gamma_{i}^{\prime \prime n}, B\right) / 2\right\},
$$

(observe that $A_{i}^{n}=\emptyset$ when $B=\emptyset$ ). Let also $\bar{\gamma}_{i}^{n}$ be an auxiliary variable which for each $\omega$ is on the segment joining $\gamma_{i}^{\prime n}$ and $\gamma_{i}^{\prime \prime n}$, and let $\widehat{\gamma}_{i}^{n}$ be 1 when $r=1$ and $1+1 / d\left(\gamma_{i}^{\prime \prime n}, B\right)$ when $r<1$. Then we set

$$
\begin{gathered}
\Phi_{i}^{n}=f\left(\gamma_{i}^{n}\right)\left(\left(k\left(\gamma_{i}^{\prime n}\right)-k\left(\gamma_{i}^{\prime \prime n}\right)\right) 1_{A_{i}^{n}}-\nabla k\left(\gamma_{i}^{\prime \prime n}\right)\left(\gamma_{i}^{\prime n}-\gamma_{i}^{\prime \prime n}\right) 1_{A_{i}^{n}}\right. \\
\left.+\left(\nabla k\left(\bar{\gamma}_{i}^{n}\right)-\nabla k\left(\gamma_{i}^{\prime \prime n}\right)\right)\left(\gamma_{i}^{\prime n}-\gamma_{i}^{\prime \prime n}\right) 1_{\left(A_{i}^{n}\right)^{c}}\right), \\
\widehat{\Phi}_{i}^{n}=f\left(\gamma_{i}^{n}\right) \nabla k\left(\gamma_{i}^{\prime \prime n}\right)\left(\gamma_{i}^{\prime n}-\gamma_{i}^{\prime \prime n}\right)
\end{gathered}
$$

(by the fact that $B$ has Lebesgue measure 0 , we see that $k$ is a.s. differentiable at the point $\gamma_{i}^{\prime \prime n}$, which is either $\beta_{i}^{n}$ or $\beta_{i}^{\prime n}$, so (7.33) and (7.34) make sense).

Lemma 7.6. Assume the following:

(i) (SH1) and (5.5) and $k$ satisfies (7.26) and (7.27);

(ii) if $r=1$ then $k$ satisfies (7.28);

(iii) if $B \neq \emptyset$ then (SH') holds;

(iv) if $r<1$ then $k$ satisfies (7.29).

(a) If $\gamma_{i}^{\prime \prime n}=\beta_{i}^{n}$ and $\gamma_{i}^{\prime n}-\gamma_{i}^{\prime \prime n}=\xi_{i}^{n}$, or if $\gamma_{i}^{\prime \prime n}=\beta_{i}^{\prime n}$ and $\gamma_{i}^{\prime n}-\gamma_{i}^{\prime \prime n}=\xi_{i}^{\prime n}$, we have for all $t>0$ :

$$
\frac{1}{\sqrt{n}} \sum_{i=1}^{[n t]} \mathbf{E}\left(\left|\Phi_{i}^{n}\right|\right) \rightarrow 0 .
$$

(b) If $\gamma_{i}^{\prime \prime n}=\beta_{i}^{n}$ and $\gamma_{i}^{\prime n}-\gamma_{i}^{\prime \prime n}=\widehat{\xi}_{i}^{n}$, or if $\gamma_{i}^{\prime \prime n}=\beta_{i}^{\prime n}$ and $\gamma_{i}^{\prime n}-\gamma_{i}^{\prime \prime n}=\widehat{\xi}_{i}^{\prime n}$, we have for all $t>0$ :

$$
\frac{1}{\sqrt{n}} \sum_{i=1}^{[n t]} \mathbf{E}\left(\left|\widehat{\Phi}_{i}^{n}\right|\right) \rightarrow 0 .
$$


Proof. 1) We first prove (7.35) when $r=1$. We choose $\varepsilon_{n}=1$ for all $n$ and putting together all estimates in (7.7), (7.8), (7.9) and (7.12) (with $l=0$, so this estimate holds for $q=2$ as well) to get

$$
q \geq 2 \Rightarrow \mathbf{E}\left(\left\|\gamma_{i}^{\prime n}-\gamma_{i}^{\prime \prime n}\right\|^{q}\right) \leq \frac{C_{q}}{n} .
$$

Then (4.7) and (7.26) and $A_{i}^{n} \subset\left\{d\left(\gamma_{i}^{\prime \prime n}, B\right)<\varepsilon\right\} \cup\left\{\left\|\gamma_{i}^{\prime n}-\gamma_{i}^{\prime \prime n}\right\| \geq \varepsilon / 2\right\}$ yield for all $A>0, \varepsilon>2 \varepsilon^{\prime}>0$ :

$$
\begin{aligned}
\left|\Phi_{i}^{n}\right|+\left|\widehat{\Phi}_{i}^{n}\right| \leq & C\left(Z_{i}^{n}\right)^{2 p}\left(\Psi_{A, \varepsilon^{\prime}}(\varepsilon)+\frac{\left\|\gamma_{i}^{\prime \prime n}\right\|}{A}\right. \\
& \left.+\left\|\gamma_{i}^{\prime n}-\gamma_{i}^{\prime \prime n}\right\|\left(\frac{1}{\varepsilon}+\frac{1}{\varepsilon^{\prime}}\right)+1_{\left\{d\left(\gamma_{i}^{\prime \prime n}, B\right) \leq \varepsilon\right\}}\right)\left\|\gamma_{i}^{\prime n}-\gamma_{i}^{\prime \prime n}\right\|
\end{aligned}
$$

If $B=\emptyset$ the indicator function above vanishes. Otherwise, the variable $\gamma_{i}^{\prime \prime n}$ has a conditional law knowing $\mathcal{F}_{\frac{i-1}{n}}$ which has a density (on $\mathbf{R}^{d}$ ) that is smaller than some (non-random) Lebesgue integrable function $\phi$ (see (3.6)), so it also has an unconditional density smaller than $\phi$. Therefore

$$
\mathbf{P}\left(d\left(\gamma_{i}^{\prime \prime n}, B\right) \leq \varepsilon\right) \leq \alpha_{\varepsilon}:=\int_{\{x: d(x, B) \leq \varepsilon\}} \phi(x) d x,
$$

and $\lim _{\varepsilon \rightarrow 0} \alpha_{\varepsilon}=0$. Then (5.5), (7.37), (7.38) and the multivariate Hölder inequality yield

$$
\mathbf{E}\left(\left|\Phi_{i}^{n}\right|\right)+\mathbf{E}\left(\left|\widehat{\Phi}_{i}^{n}\right|\right) \leq \frac{C}{\sqrt{n}}\left(\Psi_{A, \varepsilon}\left(\varepsilon^{\prime}\right)+\frac{1}{A}+\frac{1}{n^{1 / 4}}\left(\frac{1}{\varepsilon}+\frac{1}{\varepsilon^{\prime}}\right)+\alpha_{\varepsilon}^{1 / 4}\right) .
$$

Hence (7.35) readily follows: choose $A$ big, then $\varepsilon$ small, then $\varepsilon^{\prime}$ small.

2) Now we suppose that $r<1$, hence $B \neq \emptyset$. We have

$$
\begin{aligned}
& \left|\Phi_{i}^{n}\right| \leq\left(Z_{i}^{n}\right)^{2 p}\left(\left\|\gamma_{i}^{\prime n}-\gamma_{i}^{\prime \prime n}\right\|^{r} 1_{A_{i}^{n}}+\left\|\gamma_{i}^{\prime n}-\gamma_{i}^{\prime \prime n}\right\| 1_{A_{i}^{n}}\right. \\
& \left.+\frac{\left\|\gamma_{i}^{\prime n}-\gamma_{i}^{\prime \prime n}\right\|}{d\left(\gamma_{i}^{\prime \prime n}, B\right)^{1-r}} 1_{A_{i}^{n}}+\frac{\left\|\gamma_{i}^{\prime n}-\gamma_{i}^{\prime \prime n}\right\|^{2}}{d\left(\gamma_{i}^{\prime \prime n}, B\right)^{2-r}} 1_{\left(A_{i}^{n}\right)^{c}}\right) \\
& \leq C\left(Z_{i}^{n}\right)^{2 p}\left\|\gamma_{i}^{\prime n}-\gamma_{i}^{\prime \prime n}\right\|^{1+r / 2}\left(\widehat{\gamma}_{i}^{n}\right)^{1-r / 2},
\end{aligned}
$$

where the first inequality follows from (7.26), (7.27) and (7.29) for $k$, while the second one is obtained by using the definition of the set $A_{i}^{n}$. Hence Lemmas 7.4 and 7.5 readily give $(7.35)$.

3) Finally, in all cases we have

$$
\left|\widehat{\Phi}_{i}^{n}\right| \leq C\left(Z_{i}^{n}\right)^{2 p}\left\|\gamma_{i}^{\prime n}-\gamma_{i}^{\prime \prime n}\right\|\left(\widehat{\gamma}_{i}^{n}\right)^{1-r} .
$$

Therefore (7.36) follows from Lemmas 7.4 (see (7.19)) and 7.5 again. 


\section{Proof of Theorem 2.3}

1) As said at the beginning of the previous Section, we can assume that $g$ and $h$ are 1-dimensional, and that (SH1), and also (SH') when either $g$ or $h$ satisfies $\left(\mathrm{K}^{\prime}\right)$ instead of $(\mathrm{K})$, and we need to prove that the arrays defined in (7.1) and (7.1) are AN.

2) Let us prove first that $\left(\zeta_{i}^{\prime \prime n}\right)$ is AN. If $f$ is continuously differentiable, and $f$ and $\nabla f$ have polynomial growth, we readily deduce from Lebesgue's theorem that $\Sigma \mapsto \rho_{\Sigma}(f)=\mathbf{E}(f(\Sigma U))$ (where $U$ is an $\mathcal{N}\left(0, I_{d}\right)$-random vector) is bounded, continuously differentiable and with bounded derivatives over the set $\mathcal{M}^{\prime}$ defined in connection with formula (3.6). Hence if both $g$ and $h$ satisfy (K) we have (recall the notation (3.6), and set $\phi(\Sigma)=\rho_{\Sigma}(g) \rho_{\Sigma}(h)$ ):

$$
\Sigma, \Sigma^{\prime} \in \mathcal{M}^{\prime} \Rightarrow\left\{\begin{array}{l}
|\phi(\Sigma)|+\|\nabla \phi(\Sigma)\| \leq C \\
\left|\phi(\Sigma)-\phi\left(\Sigma^{\prime}\right)\right| \leq C\left\|\Sigma-\Sigma^{\prime}\right\| \\
\mid \phi(\Sigma)-\phi\left(\Sigma^{\prime}\right)-\nabla \phi\left(\Sigma^{\prime}\right)\left(\Sigma-\Sigma^{\prime}\right) \| \\
\leq \Psi\left(\| \Sigma-\Sigma^{\prime} \mid\right)\left\|\Sigma-\Sigma^{\prime}\right\|
\end{array}\right.
$$

for some constant $C$ (depending on $A_{0}$ in (3.6)) and some increasing function $\Psi$ on $\mathbf{R}_{+}$, continuous and null at 0 (here, $\nabla \phi$ is $\mathcal{M}_{d, d}$-valued, and $\nabla \phi\left(\Sigma^{\prime}\right)(\Sigma-$ $\left.\Sigma^{\prime}\right)$ is $\mathbf{R}$-valued).

If $g$ or $h$ (or both) satisfy (K') only we also have (SH'), and since

$$
\rho_{\Sigma}(f)=\int \frac{1}{(2 \pi)^{d / 2} \operatorname{det}\left(\Sigma \Sigma^{\star}\right)^{1 / 2}} f(x) \exp \left(-\frac{1}{2} x^{\star}\left(\Sigma \Sigma^{\star}\right)^{-1} x\right) d x
$$

we see that as soon as $f$ has polynomial growth the function $\Sigma \mapsto \rho_{\Sigma}(f)$ is $C^{\infty}$ with bounded derivatives of all orders on the set $\mathcal{M}^{\prime}$. Hence we also have (8.1), which thus holds in all cases.

Since we can write $(7.2)$ as $\zeta_{i}^{\prime \prime n}=\sqrt{n} \int_{(i-1) / n}^{i / n}\left(\phi\left(\sigma_{u}\right)-\phi\left(\sigma_{(i-1) / n}\right) d u\right.$, we have $\zeta_{i}^{\prime \prime n}=\eta_{i}^{n}+\eta_{i}^{\prime n}$ where

$$
\begin{gathered}
\eta_{i}^{n}=\sqrt{n} \nabla \phi\left(\sigma_{\frac{i-1}{n}}\right) \int_{\frac{i-1}{n}}^{\frac{i}{n}}\left(\sigma_{u}-\sigma_{\frac{i-1}{n}}\right) d u \\
\eta_{i}^{\prime n}=\sqrt{n} \int_{\frac{i-}{n}}^{\frac{i}{n}}\left(\phi\left(\sigma_{u}\right)-\phi\left(\sigma_{\frac{i-1}{n}}\right)-\nabla \phi\left(\sigma_{\frac{i-1}{n}}\right)\left(\sigma_{u}-\sigma_{\frac{i-1}{n}}\right)\right) d u .
\end{gathered}
$$

and we need to prove that the two arrays $\left(\eta_{i}^{n}\right)$ and $\left(\eta_{i}^{\prime n}\right)$ are AN.

We decompose further $\eta_{i}^{n}$ as $\eta_{i}^{n}=\mu_{i}^{n}+\mu_{i}^{\prime n}$, where

$$
\mu_{i}^{n}=\sqrt{n} \nabla \phi\left(\sigma_{\frac{i-1}{n}}\right) \int_{\frac{i-1}{n}}^{\frac{i}{n}} d u \int_{\frac{i-1}{n}}^{u} a_{s}^{\prime} d s
$$




$$
\begin{aligned}
\mu_{i}^{\prime n}=\sqrt{n} \nabla \phi\left(\sigma_{\frac{i-1}{n}}\right) & \int_{\frac{i-1}{n}}^{\frac{i}{n}}\left(\int_{\frac{i-1}{n}}^{u} \sigma_{s-} d W_{s}+\int_{\frac{i-1}{n}}^{u} v_{s-} d V_{s}\right. \\
& \left.+\int_{\frac{i-1}{n}}^{u} \int_{E} w(s-, x)(\mu-\nu)(d s, d x)\right) d u .
\end{aligned}
$$

On the one hand, we have $\left|\mu_{i}^{n}\right| \leq C / n^{3 / 2}$ by (8.1) and the boundedness of $a^{\prime}$, so the array $\left(\mu_{i}^{n}\right)$ is AN. On the other hand, we also get by (SH1) and (8.1) and Cauchy-Schwarz applied twice:

$$
\mathbf{E}\left(\mu_{i}^{\prime n} \mid \mathcal{F}_{\frac{i-1}{n}}\right)=0, \quad \mathbf{E}\left(\left(\mu_{i}^{\prime n}\right)^{2} \mid \mathcal{F}_{\frac{i-1}{n}}\right) \leq \frac{C}{n^{3}}
$$

Then the array $\left(\mu_{i}^{\prime n}\right)$ is $\mathrm{AN}$, as well as the array $\left(\eta_{i}^{n}\right)$.

Finally, using (8.1) once more, we see that for all $\varepsilon>0$,

$$
\begin{aligned}
\left|\eta_{i}^{\prime n}\right| & \leq \sqrt{n} \int_{\frac{i-1}{n}}^{\frac{i}{n}} \Psi\left(\left\|\sigma_{u}-\sigma_{\frac{i-1}{n}}\right\|\right)\left\|\sigma_{u}-\sigma_{\frac{i-1}{n}}\right\| d u \\
& \leq \sqrt{n} \Psi(\varepsilon) \int_{\frac{i-1}{n}}^{\frac{i}{n}}\left\|\sigma_{u}-\sigma_{\frac{i-1}{n}}\right\| d u+\frac{C \sqrt{n}}{\varepsilon} \int_{\frac{i-1}{n}}^{\frac{i}{n}}\left\|\sigma_{u}-\sigma_{\frac{i-1}{n}}\right\|^{2} d u .
\end{aligned}
$$

Since $\mathbf{E}\left(\left\|\sigma_{u}-\sigma_{\frac{i-1}{n}}\right\|^{2}\right) \leq C / n$ when $u \in((i-1) / n, i / n]$, we deduce that

$$
\sum_{i=1}^{[n t]} \mathbf{E}\left(\left|\eta_{i}^{\prime n}\right|\right) \leq C t\left(\Psi(\varepsilon)+\frac{1}{\varepsilon \sqrt{n}}\right) .
$$

From this we deduce the AN property of the array $\left(\eta_{i}^{\prime n}\right)$ because $\varepsilon>0$ is arbitrarily small and $\lim _{\varepsilon \rightarrow 0} \Psi(\varepsilon)=0$. Hence, finally, the array $\left(\zeta_{i}^{\prime \prime n}\right)$ is AN.

3) Now we start proving that the array $\left(\zeta_{i}^{\prime n}\right)$ also is AN. Since $\phi\left(\sigma_{(i-1) / n}\right)=$ $\mathbf{E}\left(g\left(\beta_{i}^{n}\right) h\left(\beta_{i}^{\prime n}\right) \mid \mathcal{F}_{(i-1) / n}\right)$, we have $\zeta_{i}^{\prime n}=\mathbf{E}\left(\delta_{i}^{n} \mid \mathcal{F}_{(i-1) / n}\right)$, where

$$
\delta_{i}^{n}=\frac{1}{\sqrt{n}}\left(g\left(\sqrt{n} \Delta_{i}^{n} Y\right) h\left(\sqrt{n} \Delta_{i+1}^{n} Y\right)-g\left(\beta_{i}^{n}\right) h\left(\beta_{i}^{\prime n}\right)\right) .
$$

Let us set

$$
\begin{gathered}
A_{i}^{n}=\left\{\left\|\sqrt{n} \Delta_{i}^{n} Y-\beta_{i}^{n}\right\|>d\left(\beta_{i}^{n}, B\right) / 2\right\}, \\
A_{i}^{\prime n}=\left\{\left\|\sqrt{n} \Delta_{i+1}^{n} Y-\beta_{i}^{\prime n}\right\|>d\left(\beta_{i}^{\prime n}, B^{\prime}\right) / 2\right\},
\end{gathered}
$$

where $B$ (resp. $B^{\prime}$ ) is either empty or is the set associated with $g$ (resp. $h$ ), according to whether that function satisfies $(\mathrm{K})$ or $\left(\mathrm{K}^{\prime}\right)$. We can express the difference $g\left(\sqrt{n} \Delta_{i}^{n} Y\right)-g\left(\beta_{i}^{n}\right)$ using a Taylor expansion if we are on the set $\left(A_{i}^{n}\right)^{c}$, and we can thus write 


$$
\begin{aligned}
& g\left(\sqrt{n} \Delta_{i}^{n} Y\right)-g\left(\beta_{i}^{n}\right) \\
&=\left(g\left(\sqrt{n} \Delta_{i}^{n} Y\right)-g\left(\beta_{i}^{n}\right)\right) 1_{A_{i}^{n}}-\nabla g\left(\beta_{i}^{n}\right)\left(\sqrt{n} \Delta_{i}^{n} Y-\beta_{i}^{n}\right) 1_{A_{i}^{n}} \\
&+\left(\nabla g\left(\bar{\gamma}_{i}^{n}\right)-\nabla g\left(\beta_{i}^{n}\right)\right)\left(\sqrt{n} \Delta_{i}^{n} Y-\beta_{i}^{n}\right) 1_{\left(A_{i}^{n}\right)^{c}} \\
&+\nabla g\left(\beta_{i}^{n}\right)\left(\sqrt{n} \Delta_{i}^{n} Y-\beta_{i}^{n}\right),
\end{aligned}
$$

where $\bar{\gamma}_{i}^{n}$ is some (random) vector lying on the segment between $\sqrt{n} \Delta_{i}^{n} Y$ and $\beta_{i}^{n}$ : recall that $\nabla g\left(\bar{\gamma}_{i}^{n}\right)$ is well defined because on $\left(A_{i}^{n}\right)^{c}$ we have $\bar{\gamma}_{i}^{n} \in B^{c}$, while $\nabla g\left(\beta_{i}^{n}\right)$ is a.s. well defined because either $B$ is empty, or it has Lebesgue measure 0 and $\beta_{i}^{n}$ has a density. Analogously, $h\left(\sqrt{n} \Delta_{i+1}^{n} Y\right)-h\left(\beta_{i}^{\prime n}\right)$ can be written likewise, provided we replace $\Delta_{i}^{n} Y, \beta_{i}^{n}, A_{i}^{n}, \bar{\gamma}_{i}^{n}$ by $\Delta_{i+1}^{n} Y, \beta_{i}^{\prime n}, A_{i}^{\prime n}$, $\bar{\gamma}_{i}^{\prime n}$.

Now observe that

$$
\begin{aligned}
\delta_{i}^{n}=\frac{1}{\sqrt{n}} g\left(\sqrt{n} \Delta_{i}^{n} Y\right)\left(h\left(\sqrt{n} \Delta_{i+1}^{n} Y\right)\right. & \left.-h\left(\beta_{i}^{\prime n}\right)\right) \\
& +\frac{1}{\sqrt{n}}\left(g\left(\sqrt{n} \Delta_{i}^{n} Y\right)-g\left(\beta_{i}^{n}\right)\right) h\left(\beta_{i}^{\prime n}\right),
\end{aligned}
$$

Therefore we deduce from the decomposition (8.2) and the analogous one for $h$, and also from (7.3) and (7.4), that $\delta_{i}^{n}=\sum_{k=1}^{6} \delta_{i}^{n}(k)$, where

$$
\begin{gathered}
\delta_{i}^{n}(1)=\frac{1}{\sqrt{n}} g\left(\sqrt{n} \Delta_{i}^{n} Y\right) \nabla h\left(\beta_{i}^{\prime n}\right) \widetilde{\xi}_{i}^{\prime \prime n}, \\
\delta_{i}^{n}(2)=\frac{1}{\sqrt{n}} g\left(\sqrt{n} \Delta_{i}^{n} Y\right) \nabla h\left(\beta_{i}^{\prime n}\right) \widetilde{\xi}_{i+1}^{n}, \\
\delta_{i}^{n}(3)=\frac{1}{\sqrt{n}} h\left(\beta_{i}^{\prime n}\right) \nabla g\left(\beta_{i}^{n}\right) \widetilde{\xi}_{i}^{n}, \\
\delta_{i}^{n}(4)=\frac{1}{\sqrt{n}}\left(g\left(\sqrt{n} \Delta_{i}^{n} Y\right) \nabla h\left(\beta_{i}^{\prime n}\right) \widehat{\xi}_{i}^{\prime n}+h\left(\beta_{i}^{\prime n}\right) \nabla g\left(\beta_{i}^{n}\right) \widehat{\xi}_{i}^{n}\right), \\
\delta_{i}^{n}(5)=\frac{1}{\sqrt{n}} g\left(\sqrt{n} \Delta_{i}^{n} Y\right)\left(\left(h\left(\sqrt{n} \Delta_{i+1}^{n} Y\right)-h\left(\beta_{i}^{\prime n}\right)\right) 1_{A_{i}^{\prime n}}\right. \\
-\nabla h\left(\beta_{i}^{\prime n}\right)\left(\sqrt{n} \Delta_{i+1}^{n} Y-\beta_{i}^{\prime n}\right) 1_{A_{i}^{\prime n}} \\
\left.+\left(\nabla h\left(\bar{\gamma}_{i}^{\prime n}\right)-\nabla h\left(\beta_{i}^{\prime n}\right)\right)\left(\sqrt{n} \Delta_{i+1}^{n} Y-\beta_{i}^{\prime n}\right) 1_{\left(A_{i}^{\prime n}\right)^{c}}\right), \\
\delta_{i}^{n}(6)=\frac{1}{\sqrt{n}} h\left(\beta_{i}^{\prime n}\right)\left(\left(g\left(\sqrt{n} \Delta_{i}^{n} Y\right)-g\left(\beta_{i}^{n}\right)\right) 1_{A_{i}^{n}}\right. \\
\quad-\nabla g\left(\beta_{i}^{n}\right)\left(\sqrt{n} \Delta_{i}^{n} Y-\beta_{i}^{n}\right) 1_{A_{i}^{n}} \\
\left.+\left(\nabla g\left(\bar{\gamma}_{i}^{n}\right)-\nabla g\left(\beta_{i}^{n}\right)\right)\left(\sqrt{n} \Delta_{i}^{n} Y-\beta_{i}^{n}\right) 1_{\left(A_{i}^{n}\right)^{c}}\right) .
\end{gathered}
$$


If we combine (5.2) with Lemma 7.6, we readily get $\sum_{i=1}^{[n t]} \mathbf{E}\left(\left\|\delta_{i}^{n}(k)\right\|\right) \rightarrow 0$ when $k=4,5,6$. So we are left to proving that

$$
\text { the array }\left\{\mu_{i}^{n}(k)=\mathbf{E}\left(\delta_{i}^{n}(k) \mid \mathcal{F}_{\frac{i-1}{n}}\right)\right\} \text { is AN. }
$$

for $k=1,2,3$.

4) Let us introduce the $\mathcal{M}_{d, d^{\prime}}$-valued martingales

$$
M(n, i)_{t}= \begin{cases}0 & \text { if } t \leq \frac{i-1}{n} \\ v_{\frac{i-1}{n}}\left(V_{t}-V_{\frac{i-1}{n}}\right)+\int_{\frac{i-1}{n}}^{t} \int_{E_{n}} w\left(\frac{i-1}{n}, x\right)(\mu-\nu)(d s, d x) & \text { otherwise. }\end{cases}
$$

We see that $\widetilde{\xi}_{i}^{n}=\zeta(2)_{i}^{n}+\zeta(5)_{i}^{n}+\zeta(7)_{i}^{n}=\sqrt{n}\left(\eta_{i}^{n}+\eta_{i}^{\prime n}\right)$, where

$$
\begin{gathered}
\eta_{i}^{n}=\frac{1}{n} a_{\frac{i-1}{n}}+\int_{\frac{i-1}{n}}^{\frac{i}{n}}\left(W_{u}-W_{\frac{i-1}{n}}\right) d W_{u}, \\
\eta_{i}^{\prime n}=\int_{\frac{i-1}{n}}^{\frac{i}{n}} M(n, i)_{u} d W_{u}=\Delta_{i}^{n} M(n, i) \Delta_{i}^{n} W-\int_{\frac{i-1}{n}}^{\frac{i}{n}} d M(n, i)_{u} W_{u} .
\end{gathered}
$$

Now we can write

$$
\mu_{i}^{n}(3)=\rho_{i-1}^{n}(h) \mathbf{E}\left(\nabla g\left(\sqrt{n} \sigma_{\frac{i-1}{n}} \Delta_{i}^{n} W\right)\left(\eta_{i}^{n}+\eta_{i}^{\prime n}\right) \mid \mathcal{F}_{\frac{i-1}{n}}\right) .
$$

$g$ is even, so $\nabla g$ is odd; hence the variable $\nabla g\left(\sqrt{n} \sigma_{\frac{i-1}{n}} \Delta_{i}^{n} W\right) \eta_{i}^{n}$ is multiplied by -1 if we change the sign of the process $\left(W_{s}-\stackrel{n}{W}_{(i-1) / n}\right)_{s \geq(i-1) / n}$, and this sign change does not affect the $\mathcal{F}_{(i-1) / n}$-conditional distribution of this process. Hence we get

$$
\mathbf{E}\left(\nabla g\left(\sqrt{n} \sigma_{\frac{i-1}{n}} \Delta_{i}^{n} W\right) \eta_{i}^{n} \mid \mathcal{F}_{\frac{i-1}{n}}\right)=0
$$

On the other hand, the processes $M(n, i)$ and $W_{s}-W_{(i-1) / n}$ are independent, conditionally on $\mathcal{F}_{(i-1) / n}$, when the times goes through $((i-$ $1) / n, i / n]$. So if $\mathcal{F}_{s}^{0}$ denotes the $\sigma$-field generated by $\mathcal{F}_{(i-1) / n}$ and by $\left(W_{u}-W_{(i-1) / n}\right)_{(i-1) / n \leq u \leq s}$, we get that $M(n, i)$ is an $\left(\mathcal{F}_{s}^{0}\right)$-martingale for $s \in((i-1) / n, i / n]$, and thus $\mathbf{E}\left(\eta_{i}^{\prime n} \mid \mathcal{F}_{i / n}^{0}\right)=0$. By successive conditioning, we immediately deduce that

$$
\mathbf{E}\left(\nabla g\left(\sqrt{n} \sigma_{\frac{i-1}{n}} \Delta_{i}^{n} W\right) \eta_{i}^{\prime n}|| \mathcal{F}_{\frac{i-1}{n}}\right)=0,
$$

and therefore $\mu_{i}^{n}(3)=0$. In a similar way, $\nabla h$ is odd and $\beta_{i}^{\prime n}$ is the product of an $\mathcal{F}_{(i-1) / n}$-measurable variable, times $\Delta_{i+1}^{n} W$. So exactly as above we have

$$
\mathbf{E}\left(\nabla h\left(\beta_{i}^{\prime n}\right) \widetilde{\xi}_{i+1}^{n} \mid \mathcal{F}_{\frac{i}{n}}\right)=0
$$


and so a fortiori $\mu_{i}^{n}(2)=0$.

5) It remains to study $\mu_{i}^{n}(1)$. With the previous notation $M(n, i)$, it is easy to check that

$\left.\mu_{i}^{n}(1)=\frac{1}{\sqrt{n}} \sum_{l=1}^{d} \sum_{m=1}^{d^{\prime}} z_{i}^{n, l m} \mathbf{E}\left(g\left(\sqrt{n} \Delta_{i}^{n} Y\right)\left(\sigma_{\frac{i-1}{n}}^{\prime} \Delta_{i}^{n} W+\Delta_{i}^{n} M(n, i)\right)^{l m}\right) \mid \mathcal{F}_{\frac{i-1}{n}}\right)$,

where $z_{i}^{n, l m}=\int \partial_{x_{l}} h\left(\sigma_{\frac{i-1}{n}} x\right) x_{m} \rho(d x)$ and $\rho$ is $\mathcal{N}\left(0, I_{d^{\prime}}\right)$ (the law of $\left.W_{1}\right)$, so $\left\|z_{i}^{n, l m}\right\| \leq C$. Recalling once more $\sqrt{n} \Delta_{i}^{n} Y=\beta_{i}^{n}+\widehat{\xi}_{i}^{n}+\widetilde{\xi}_{i}^{n}$, we see that

$$
\mu_{i}^{n}(1)=\sum_{l=1}^{d} \sum_{m=1}^{d^{\prime}}\left(\mathbf{E}\left(\mu_{i}^{n}(l, m) \mid \mathcal{F}_{\frac{i-1}{n}}\right)+\mathbf{E}\left(\mu_{i}^{\prime n}(l, m) \mid \mathcal{F}_{\frac{i-1}{n}}\right)\right),
$$

where

$$
\begin{gathered}
\mu_{i}^{n}(l, m)=\frac{1}{\sqrt{n}} z_{i}^{n, l m}\left(g\left(\beta_{i}^{n}+\widehat{\xi}_{i}^{n}+\widetilde{\xi}_{i}^{n}\right)-g\left(\beta_{i}^{n}\right)\right)\left(\sigma_{\frac{i-1}{n}}^{\prime} \Delta_{i}^{n} W+\Delta_{i}^{n} M(n, i)\right)^{l m}, \\
\mu_{i}^{\prime n}(l, m)=\frac{1}{\sqrt{n}} z_{i}^{n, l m} g\left(\beta_{i}^{n}\right)\left(\sigma_{\frac{i-1}{n}}^{\prime} \Delta_{i}^{n} W+\Delta_{i}^{n} M(n, i)\right)^{l m} .
\end{gathered}
$$

Use (5.2) and (7.37) and the property $\mathbf{E}\left(\left\|\Delta_{i}^{n} W\right\|^{q}\right)+\mathbf{E}\left(\left\|\Delta_{i}^{n} M(n, i)\right\|^{q}\right) \leq$ $C_{q} / n$ for all $q \geq 2$ to get that $\sum_{i=1}^{[n t]} \mathbf{E}\left(\left|\mu_{i}^{n}(l, m)\right|\right) \rightarrow 0$. Finally, since $g$ is even and $\Delta_{i}^{n} W$ and $\Delta_{i}^{n} M(n, i)$ are independent conditionally on $\mathcal{F}_{(i-1) / n}$ and $\mathbf{E}\left(\Delta_{i}^{n} M(n, i) \mid \mathcal{F}_{(i-1) / n}\right)=0$, we find that indeed $\mathbf{E}\left(\mu_{i}^{\prime n}(l, m) \mid \mathcal{F}_{(i-1) / n}\right)=0$. So we get (8.3) for $k=1$, and we are done.

\section{References}

1. Andersen T.G., Bollerslev T., Diebold F.X. and Labys P. (2003): Modeling and Forecasting realized Volatility. Econometrica, 71, 579-625.

2. Andersen T.G., Bollerslev T. and Diebold F.X. (2004): Parametric and nonparametric measurement of volatility, in "Handbook of Financial Econometrics", edited by Ait-Sahalia Y. and Hansen L.P., North Holland, Amsterdam, Forthcoming.

3. Barndorff-Nielsen O.E. and Shephard N. (2002): Econometric analysis of realised volatility and its use in estimating stochastic volatility models. Journal of the Royal Statistical Society, Series B, 64, 253-280.

4. Barndorff-Nielsen O.E. and Shephard N. (2003): Econometrics of testing for jumps in financial economics using bipower variation. Unpublished discussion paper: Nuffield College, Oxford.

5. Barndorff-Nielsen O.E. and Shephard N. (2004): Power and bipower variation with stochastic volatility and jumps (with discussion). Journal of Financial Econometrics, 2, 1-48. 
6. Barndorff-Nielsen O.E. and Shephard N. (2004): Econometric analysis of realised covariation: high frequency covariance, regression and correlation in financial economics. Econometrica, 72, 885-925.

7. Becker E. (1998): Thórèmes limites pour des processus discrétisés. PhD Thesis, Univ. P. et M. Curie.

8. Jacod, J. (1994): Limit of random measures associated with the increments of a Brownian semimartingale. Unpublished manuscript.

9. Jacod, J. and Shiryaev, A. (2003): Limit Theorems for Stochastic Processes, 2d edition. Springer-Verlag: Berlin.

10. Jacod, J. (2004): Inference for stochastic processes, in "Handbook of Financial Econometrics", edited by Ait-Sahalia Y. and Hansen L.P., North Holland, Amsterdam, Forthcoming.

11. Rényi A. (1963): On stable sequences of events. Sankhya, Ser. A, 25, 293-302. 